\title{
Enantioselective Protonation of Catalytically Generated Chiral Enolates as an Approach to the Synthesis of $\alpha$-Chloroesters.
}

\author{
Nathan T. Reynolds and Tomislav Rovis* \\ Department of Chemistry, Colorado State University \\ Fort Collins, Colorado 80523
}

\section{Supporting Information}

General Methods. All reactions were carried out under an atmosphere of argon in flame-dried glassware with magnetic stirring. Toluene was degassed with argon and passed through one column of neutral alumina and one column of Q5 reactant. Column chromatography was performed on EM Science silica gel 60 (230-400 mesh). Thin layer chromatography was performed on EM Science $0.25 \mathrm{~mm}$ silica gel 60-F plates. Visualization was accomplished with UV light, $\mathrm{KMnO}_{4}$, or aqueous ceric ammonium molybdate dips followed by heating.

Phenols were purchased from Aldrich Chemical Co. and used after azeotropic drying with toluene. Aldehydes were obtained from Aldrich Chemical Co. or synthesized from the corresponding alcohol. $\alpha-\alpha$-Dihaloaldehydes were prepared by an adaptation of literature procedures. ${ }^{1}$ Previously synthesized esters matched reported spectra and new esters were characterized below.

Infrared spectra were obtained on a Nicolet Avatar 320 FT-IR spectrometer. ${ }^{1} \mathrm{H}$ NMR and spectra were recorded on a Varian 300 or $400 \mathrm{MHz}$ spectrometer at ambient temperature. Data are reported as follows: chemical shift in parts per million $(\delta, \mathrm{ppm})$ from an internal standard [tetramethylsilane (TMS)] or deuterated chloroform $\left(\mathrm{CDCl}_{3}\right)$, multiplicity $(\mathrm{s}=$ singlet, $\mathrm{d}=$ doublet, $\mathrm{t}=$ triplet, $\mathrm{q}=$ quartet, and $\mathrm{m}=$ multiplet $)$, integration, and coupling constant $(\mathrm{Hz}) .{ }^{13} \mathrm{C}$ NMR were recorded on a Varian 300, or 400 $\mathrm{MHz}$ spectrometer at ambient temperature. Chemical shifts are reported in ppm from $\left(\mathrm{CDCl}_{3}\right)$ taken as $77.0 \mathrm{ppm}$. Mass spectra were obtained on Fisons VG Autospec. Analytical high performance liquid chromatography (HPLC) was performed on an Agilent 1100 series HPLC equipped with a diode array detector using Chiracel chiral columns as indicated.

General procedure for the synthesis of $\alpha$-halo-esters: A flame-dried round bottom was charged with potassium hydride (1.0 equiv.) and 18-crown-6 (0.5 equiv.). Toluene (0.062 M), 2,6-dibromo-4-methylphenol (1.2 equiv.), and phenol (10.0 equiv.) were added via syringe and the solution was stirred at ambient temperature for 5 minutes. Triazolium salt ( 0.10 equiv.) was added and the solution stirred for an additional 5 minutes. $\alpha$ - $\alpha$-dihaloaldehyde was then added via syringe and the reaction was stirred at room temperature for 19 hours. The reaction mixture was then filtered through a pad of silica gel, eluting with diethyl ether. After washing with $1 \mathrm{M} \mathrm{NaOH} \mathrm{(2x),} \mathrm{brine} \mathrm{(1x),} \mathrm{and}$ drying over $\mathrm{MgSO}_{4}$, the solution was concentrated onto silica gel. Flash column chromatography (97:3 hexane:ethyl ether) afforded the desired product. 


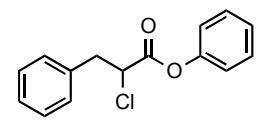

2-Chloro-3-phenyl-propionic acid phenyl ester: $\mathrm{Rf}=0.58(3: 1$ Hex/EtOAc $) ;[\alpha]_{\mathrm{D}}{ }^{24}=+38.8\left(\mathrm{c}=2.4, \mathrm{CHCl}_{3}\right) ;$ HPLC analysis Chiracel OB-H column 97:3 hexanes : isopropanol $0.3 \mathrm{~mL} / \mathrm{min}$. Major: 38.0 minutes; Minor: 42.7 minutes. ${ }^{1} \mathrm{H}$ NMR $\left(400 \mathrm{MHz}, \mathrm{CDCl}_{3}\right) \delta$ 7.25-7.39 (m, $8 \mathrm{H}), 6.97(\mathrm{~d}, 2 \mathrm{H}, J=7.7 \mathrm{~Hz}), 4.68(\mathrm{dd}, 1 \mathrm{H}, J=7.7,7.7 \mathrm{~Hz}), 3.49(\mathrm{dd}, 1 \mathrm{H}, J=7.9,13.9$ $\mathrm{Hz}), 3.33(\mathrm{dd}, 1 \mathrm{H}, J=7.2,13.9 \mathrm{~Hz}) ;{ }^{13} \mathrm{C} \mathrm{NMR}\left(100 \mathrm{MHz}, \mathrm{CDCl}_{3}\right) \delta 167.7,150.2,135.5$, 129.5, 129.4, 128.7, 127.6, 126.3, 121.0, 57.0, 41.1: IR (NaCl, neat), 1766, 1592, 1493, 1456, $1192 \mathrm{~cm}^{-1}$; HRMS (FAB+) calcd for $\mathrm{C}_{15} \mathrm{H}_{13} \mathrm{ClO}_{2}, 261.0682$. Found 261.0688.

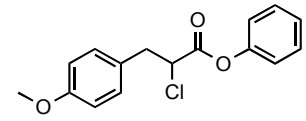

2-Chloro-3-(4-methoxy-phenyl)-propionic acid phenyl ester: $\mathrm{Rf}=$ $0.48(3: 1 \mathrm{Hex} / \mathrm{EtOAc}) ;[\alpha]_{\mathrm{D}}{ }^{24}=+27.3\left(\mathrm{c}=5.5, \mathrm{CHCl}_{3}\right) ;$ HPLC analysis - Chiracel AD-H column $99: 1$ hexanes : isopropanol $0.3 \mathrm{~mL}$ / min. Major: 39.0 minutes, Minor: 35.7 minutes. ${ }^{1} \mathrm{H}$ NMR $\left(400 \mathrm{MHz}, \mathrm{CDCl}_{3}\right) \delta 7.38$ $(\mathrm{dd}, 2 \mathrm{H}, J=7.5,7.5 \mathrm{~Hz}), 7.21-7.27(\mathrm{~m}, 3 \mathrm{H}), 7.00(\mathrm{~d}, 2 \mathrm{H}, J=7.5 \mathrm{~Hz}), 6.90(\mathrm{~d}, 2 \mathrm{H}, J=8.7$ $\mathrm{Hz}), 4.63(\mathrm{dd}, 1 \mathrm{H}, J=7.0,7.9 \mathrm{~Hz}), 3.82(\mathrm{~s}, 3 \mathrm{H}), 3.44(\mathrm{dd}, 1 \mathrm{H}, J=7.9,14.1 \mathrm{~Hz}), 3.28(\mathrm{dd}$, $1 \mathrm{H}, J=7.0,14.1 \mathrm{~Hz}) ;{ }^{13} \mathrm{C} \mathrm{NMR}\left(100 \mathrm{MHz}, \mathrm{CDCl}_{3}\right) \quad \delta 167.8,159.0,150.2,130.5,129.5$, 127.5, 126.3, 121.0, 114.1, 57.2, 55.2, 40.3; IR (NaCl, neat), 1766, 1612, 1514, 1250, $1191 \mathrm{~cm}^{-1}$; HRMS (FAB+) calcd for $\mathrm{C}_{16} \mathrm{H}_{16} \mathrm{ClO}_{3}, 291.0788$. Found 291.0778.

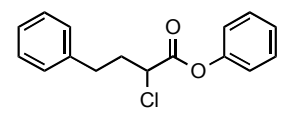

2-Chloro-4-phenyl-butyric acid phenyl ester: $\quad \mathrm{Rf}=0.56(3: 1$ Hex/EtOAc $) ;[\alpha]_{\mathrm{D}}{ }^{24}=-6.1\left(\mathrm{c}=4.1, \mathrm{CHCl}_{3}\right) ; \quad$ HPLC analysis Chiracel OD-H column $97: 3$ hexanes : isopropanol $1.0 \mathrm{~mL} / \mathrm{min}$. Major: 7.7 minutes, Minor: 8.6 minutes. ${ }^{1} \mathrm{H}$ NMR $\left(300 \mathrm{MHz}, \mathrm{CDCl}_{3}\right) \delta$ 7.25-7.44 (m, $8 \mathrm{H}), 7.12(\mathrm{~d}, 2 \mathrm{H}, J=7.7 \mathrm{~Hz}), 4.47(\mathrm{dd}, 1 \mathrm{H}, J=5.5,8.4 \mathrm{~Hz}), 2.83-3.00(\mathrm{~m}, 2 \mathrm{H}), 2.34-$ $2.57(\mathrm{~m}, 2 \mathrm{H}) ;{ }^{13} \mathrm{C} \mathrm{NMR}\left(75 \mathrm{MHz}, \mathrm{CDCl}_{3}\right) \delta 167.9,150.2,139.6,129.4,128.6,128.5$, 126.4, 126.2, 121.0, 56.4, 36.3, 32.1; IR (NaCl, neat), 1765, 1592, 1493, 1192, $1163 \mathrm{~cm}^{-}$ ${ }^{1}$; HRMS (FAB+) calcd for $\mathrm{C}_{16} \mathrm{H}_{16} \mathrm{ClO}_{2}, 275.0839$. Found 275.0826.

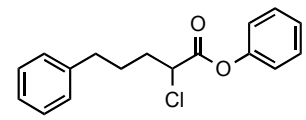

2-Chloro-5-phenyl-pentanoic acid phenyl ester: $\mathrm{Rf}=0.58(3: 1$ $\mathrm{Hex} /$ EtOAc $) ;[\alpha]_{\mathrm{D}}{ }^{24}=-3.4\left(\mathrm{c}=4.8, \mathrm{CHCl}_{3}\right) ;$ HPLC analysis Chiracel OJ-H column 90 : 10 hexanes : isopropanol $1.0 \mathrm{~mL} / \mathrm{min}$. Major: 32.2 minutes, Minor: 20.2 minutes. ${ }^{1} \mathrm{H}$ NMR $\left(400 \mathrm{MHz}, \mathrm{CDCl}_{3}\right) \delta 7.41(\mathrm{dd}, 2 \mathrm{H}, J$ $=7.7 \mathrm{~Hz}), 7.21-7.34(\mathrm{~m}, 6 \mathrm{H}), 7.11(\mathrm{~d}, 2 \mathrm{H}, J=7.9 \mathrm{~Hz}), 4.51(\mathrm{dd}, 1 \mathrm{H}, J=6.2,7.9 \mathrm{~Hz})$, 2.73 (ddd, $2 \mathrm{H}, J=1.9,8.5,8.5 \mathrm{~Hz}), 2.07-2.29(\mathrm{~m}, 2 \mathrm{H}), 1.81-1.99(\mathrm{~m}, 2 \mathrm{H}) ;{ }^{13} \mathrm{C}$ NMR $(100$ $\left.\mathrm{MHz}_{\mathrm{CDCl}}\right) \delta 168.1,150.3,141.2,129.5,128.5,128.4,126.3,126.1,121.1,57.0,35.0$, 34.2, 27.7; IR (NaCl, neat) 1764, 1493, 1192, 1163, $1135 \mathrm{~cm}^{-1}$; HRMS (FAB+) calcd for $\mathrm{C}_{17} \mathrm{H}_{18} \mathrm{ClO}_{2}, 289.0995$. Found 289.0986.

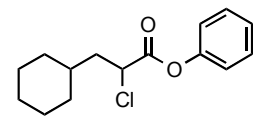

2-Chloro-3-cyclohexyl-propionic acid phenyl ester: $\mathrm{Rf}=0.64$ (3:1 Hex/EtOAc); $[\alpha]_{\mathrm{D}}{ }^{24}=-13.6\left(\mathrm{c}=1.6, \mathrm{CHCl}_{3}\right) ;$ HPLC analysis Chiracel OJ-H column $97: 3$ hexanes : isopropanol $1 \mathrm{~mL} / \mathrm{min}$. Major: 10.5 minutes, Minor: 8.4 minutes. ${ }^{1} \mathrm{H}$ NMR $\left(400 \mathrm{MHz}, \mathrm{CDCl}_{3}\right) \delta 7.41(\mathrm{dd}, 2 \mathrm{H}, J=7.7$, $7.7 \mathrm{~Hz}), 7.26(\mathrm{dd}, 1 \mathrm{H}, J=7.5,7.5 \mathrm{~Hz}), 7.12(\mathrm{~d}, 2 \mathrm{H}, J=7.7 \mathrm{~Hz}), 4.57(\mathrm{dd}, 1 \mathrm{H}, J=6.4,8.5$ $\mathrm{Hz}), 1.93-2.06(\mathrm{~m}, 2 \mathrm{H}), 1.67-1.82(\mathrm{~m}, 5 \mathrm{H}), 1.58-1.63(\mathrm{~m}, 1 \mathrm{H}), 1.13-1.34(\mathrm{~m}, 3 \mathrm{H}), 0.87-$ $1.08(\mathrm{~m}, 2 \mathrm{H}) ;{ }^{13} \mathrm{C} \mathrm{NMR}\left(100 \mathrm{MHz}, \mathrm{CDCl}_{3}\right) \quad \delta 168.65,150.4,129.5,126.3,121.1,55.2$, 
42.1, 34.5, 33.3, 32.2, 26.3, 26.1, 25.9; IR (NaCl, neat) 1767, 1493, 1192, 1163, 1142 $\mathrm{cm}^{-1}$; HRMS (FAB+) calcd for $\mathrm{C}_{15} \mathrm{H}_{20} \mathrm{ClO}, 267.1152$. Found 267.1139.

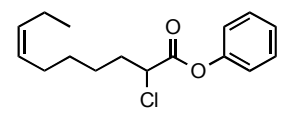

2-Chloro-dec-7-enoic acid phenyl ester: $\mathrm{Rf}=0.67(3: 1 \mathrm{Hex} / \mathrm{EtOAc})$; $[\alpha]_{\mathrm{D}}{ }^{24}=-8.0\left(\mathrm{c}=2.5, \mathrm{CHCl}_{3}\right) ;$ HPLC analysis - Chiracel OJ-H column $97: 3$ hexanes : isopropanol $1 \mathrm{~mL} / \mathrm{min}$. Major: 11.4 minutes, Minor: 8.3 minutes. ${ }^{1} \mathrm{H}$ NMR $\left(400 \mathrm{MHz}, \mathrm{CDCl}_{3}\right) \delta 7.41(\mathrm{dd}, 2 \mathrm{H}, J=7.5,7.5 \mathrm{~Hz}), 7.25$ $7.29(\mathrm{~m}, 1 \mathrm{H}), 7.12(\mathrm{dd}, 2 \mathrm{H}, J=1.1,8.7 \mathrm{~Hz}), 5.37-5.44(\mathrm{~m}, 1 \mathrm{H}), 5.29-5.36(\mathrm{~m}, 1 \mathrm{H}), 4.49$ $(\mathrm{dd}, 1 \mathrm{H}, J=6.2,8.2 \mathrm{~Hz}), 2.14-2.23(\mathrm{~m}, 1 \mathrm{H}), 2.01-2.12(\mathrm{~m}, 5 \mathrm{H}), 1.50-1.63(\mathrm{~m}, 2 \mathrm{H}), 1.41-$ $1.49(\mathrm{~m}, 2 \mathrm{H}), 0.97(\mathrm{t}, 3 \mathrm{H}, J=7.7 \mathrm{~Hz}) ;{ }^{13} \mathrm{C} \mathrm{NMR}\left(100 \mathrm{MHz}, \mathrm{CDCl}_{3}\right) \quad \delta 168.3,150.4$, 132.2, 129.5, 128.4, 126.3, 121.1, 57.1, 34.7, 28.9, 26.7, 25.6, 20.5, 14.3; IR (NaCl, neat) 1768, 1493, 1458, 1193, $1163 \mathrm{~cm}^{-1}$; HRMS (FAB+) calcd for $\mathrm{C}_{16} \mathrm{H}_{22} \mathrm{ClO}_{2}, 281.1308$. Found 281.1307.

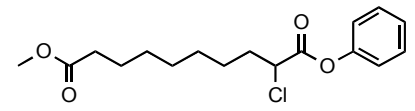

2-Chloro-decanedioic acid 10-methyl ester 1-phenyl ester: $\mathrm{Rf}=0.36(3: 1 \mathrm{Hex} / \mathrm{EtOAc}) ;[\alpha]_{\mathrm{D}}{ }^{24}=-5.2 \quad\left(\mathrm{c}=5.8, \mathrm{CHCl}_{3}\right)$; HPLC analysis - Chiracel AD-H column $97: 3$ hexanes : isopropanol $1 \mathrm{~mL} / \mathrm{min}$. Major: 8.7 minutes, Minor: 8.2 minutes. ${ }^{1} \mathrm{H}$ NMR $(400 \mathrm{MHz}$, $\left.\mathrm{CDCl}_{3}\right) \delta 7.38(\mathrm{dd}, 2 \mathrm{H}, J=7.7,7.7 \mathrm{~Hz}), 7.24(\mathrm{dd}, 1 \mathrm{H}, J=7.5,7.5 \mathrm{~Hz}), 7.10(\mathrm{~d}, 2 \mathrm{H}, J=$ $7.7 \mathrm{~Hz}), 4.46(\mathrm{dd}, 1 \mathrm{H}, J=6.2,8.1 \mathrm{~Hz}), 3.64(\mathrm{~s}, 3 \mathrm{H}), 2.29(\mathrm{t}, 2 \mathrm{H}, J=7.5 \mathrm{~Hz}), 2.09-2.18$ $(\mathrm{m}, 1 \mathrm{H}), 1.98-2.07(\mathrm{~m}, 1 \mathrm{H}), 1.42-1.64(\mathrm{~m}, 4 \mathrm{H}), 1.33(\mathrm{~m}, 6 \mathrm{H}) ;{ }^{13} \mathrm{C}$ NMR $(100 \mathrm{MHz}$, $\left.\mathrm{CDCl}_{3}\right) \delta 174.2,168.3,150.3,129.5,126.3,121.1,57.1,51.5,34.7,34.0,28.9,28.6,25.9$, 24.8; IR ( $\mathrm{NaCl}$, neat) 1766, 1737, 1493, 1193, $1163 \mathrm{~cm}^{-1}$; HRMS (FAB+) calcd for $\mathrm{C}_{17} \mathrm{H}_{24} \mathrm{ClO}_{4}, 327.13630$. Found 327.1346.

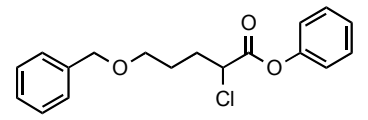

5-Benzyloxy-2-chloro-pentanoic acid phenyl ester: $\mathrm{Rf}=0.54$ $(3: 1 \mathrm{Hex} / \mathrm{EtOAc}) ;[\alpha]_{\mathrm{D}}{ }^{24}=-6.3\left(\mathrm{c}=3.8, \mathrm{CHCl}_{3}\right) ; \mathrm{HPLC}$ analysis - Chiracel OD-H column $97: 3$ hexanes : isopropanol $1 \mathrm{~mL} / \mathrm{min}$. Major: 12.0 minutes, Minor: 13.8 minutes. ${ }^{1} \mathrm{H} \mathrm{NMR}\left(400 \mathrm{MHz}, \mathrm{CDCl}_{3}\right) \delta 7.38(\mathrm{dd}, 2 \mathrm{H}$, $J=7.7,7.7 \mathrm{~Hz}), 7.33-7.34(\mathrm{~m}, 4 \mathrm{H}), 7.23-7.31(\mathrm{~m}, 2 \mathrm{H}), 7.1(\mathrm{dd}, 2 \mathrm{H}, J=8.1,8.1 \mathrm{~Hz}), 4.54$ $(\mathrm{dd}, 1 \mathrm{H}, J=5.8,8.1 \mathrm{~Hz}), 4.51(\mathrm{~s}, 2 \mathrm{H}), 3.55(\mathrm{t}, 2 \mathrm{H}, J=6.2 \mathrm{~Hz}), 2.28-2.36(\mathrm{~m}, 1 \mathrm{H}), 2.11-$ $2.20(\mathrm{~m}, 1 \mathrm{H}), 1.79-1.94(\mathrm{~m}, 2 \mathrm{H}) ;{ }^{13} \mathrm{C} \mathrm{NMR}\left(100 \mathrm{MHz}, \mathrm{CDCl}_{3}\right) \delta 168.2,150.3,138.2$, 129.5, 128.4, 127.6, 126.3, 121.1, 72.9, 69.0, 57.0, 31.8, 26.2; IR (NaCl, neat) 1765, 1493, 1193, 1163, $1144 \mathrm{~cm}^{-1}$; HRMS (FAB+) calcd for $\mathrm{C}_{18} \mathrm{H}_{20} \mathrm{ClO}_{3}, 319.1101$. Found 319.1095 .

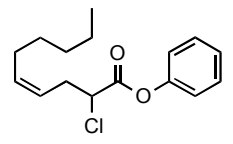

2-Chloro-dec-4-enoic acid phenyl ester: $\mathrm{Rf}=0.70(3: 1 \mathrm{Hex} / \mathrm{EtOAc})$; $[\alpha]_{\mathrm{D}}{ }^{24}=-6.6\left(\mathrm{c}=3.9, \mathrm{CHCl}_{3}\right) ; \mathrm{HPLC}$ analysis - Chiracel OJ-H column 97 : 3 hexanes : isopropanol $1 \mathrm{~mL} / \mathrm{min}$. Major: 7.7 minutes, Minor: 6.6 minutes. ${ }^{1} \mathrm{H}$ NMR $\left(400 \mathrm{MHz}, \mathrm{CDCl}_{3}\right) \delta 7.40(\mathrm{dd}, 2 \mathrm{H}, J=7.9,7.9 \mathrm{~Hz}), 7.26(\mathrm{dd}, 2 \mathrm{H}, J=$ 7.5, $7.5 \mathrm{~Hz}), 7.12(\mathrm{dd}, 2 \mathrm{H}, J=1.1,8.7 \mathrm{~Hz}), 5.63-5.69(\mathrm{~m}, 1 \mathrm{H}), 5.42-5.48(\mathrm{~m}, 1 \mathrm{H}), 4.49$ $(\mathrm{dd}, 1 \mathrm{H}, J=7.0,7.0 \mathrm{~Hz}), 2.94(\mathrm{ddd}, 1 \mathrm{H}, J=14.3,7.0,7.6 \mathrm{~Hz}), 2.83$ (ddd, 1H, $J=14.4$, 7.2, 7.6 Hz), 2.10 (ddd, $2 \mathrm{H}, J=7.6,7.2,7.2 \mathrm{~Hz}), 1.27-1.42(\mathrm{~m}, 6 \mathrm{H}), 0.89$ (t, $3 \mathrm{H}, J=6.8$ $\mathrm{Hz}) ;{ }^{13} \mathrm{C} \mathrm{NMR}\left(100 \mathrm{MHz}, \mathrm{CDCl}_{3}\right) \delta 167.9,150.4,135.1,129.5,126.3,122.2,121.1,56.3$, 32.9, 31.4, 29.1, 27.5, 22.5, 14.0; IR (NaCl, neat) 1768, 1493, 1193, 1163, $1142 \mathrm{~cm}^{-1}$; HRMS (FAB+) calcd for $\mathrm{C}_{16} \mathrm{H}_{22} \mathrm{ClO}_{2}, 281.1308$. Found 281.1297. 


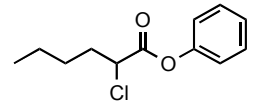

2-Chloro-hexanoic acid phenyl ester: $\mathrm{Rf}=0.64(3: 1 \mathrm{Hex} / \mathrm{EtOAc})$; $[\alpha]_{\mathrm{D}}{ }^{24}=-11.4\left(\mathrm{c}=2.6, \mathrm{CHCl}_{3}\right) ; \mathrm{HPLC}$ analysis - Chiracel OJ-H column 97 : 3 hexanes : isopropanol $1 \mathrm{~mL} /$ min. Major: 13.3 minutes, Minor: 9.8 minutes. ${ }^{1} \mathrm{H}$ NMR $\left(300 \mathrm{MHz}, \mathrm{CDCl}_{3}\right) \delta$ 7.37-7.43 (m, 2H), 7.24-7.29 (m, 1H), 7.11$7.15(\mathrm{~m}, 2 \mathrm{H}), 4.49(\mathrm{dd}, 1 \mathrm{H}, J=6.3,7.8 \mathrm{~Hz}), 2.01-2.24(\mathrm{~m}, 2 \mathrm{H}), 1.36-1.62(\mathrm{~m}, 4 \mathrm{H}), 0.96$ $(\mathrm{t}, 3 \mathrm{H}, J=7.2 \mathrm{~Hz}) ;{ }^{13} \mathrm{C} \mathrm{NMR}\left(75 \mathrm{MHz}, \mathrm{CDCl}_{3}\right) \delta 168.3,150.4,129.5,126.3,121.1,57.2$, 34.5, 28.1, 22.0, 13.8; IR ( NaCl, neat) 1768, 1493, 1193, 1163, $1144 \mathrm{~cm}^{-1}$; HRMS $(\mathrm{FAB}+)$ calcd for $\mathrm{C}_{12} \mathrm{H}_{16} \mathrm{ClO}_{2}, 227.0839$. Found 227.0834.

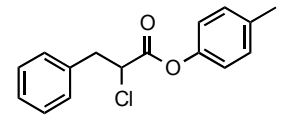

2-Chloro-3-phenyl-propionic acid $\boldsymbol{p}$-tolyl ester: $\quad \mathrm{Rf}=0.59(3: 1$ $\mathrm{Hex} / \mathrm{EtOAc}) ;[\alpha]_{\mathrm{D}}{ }^{24}=+28.9\left(\mathrm{c}=2.0, \mathrm{CHCl}_{3}\right) ;$ HPLC analysis Chiracel AD-H column 97 : 3 hexanes : isopropanol $1 \mathrm{~mL} / \mathrm{min}$. Major: 24.8 minutes, Minor: 23.3 minutes. ${ }^{1} \mathrm{H}$ NMR $\left(400 \mathrm{MHz}, \mathrm{CDCl}_{3}\right) \delta$ 7.29-7.39 (m, $5 \mathrm{H}), 7.15-7.17(\mathrm{~m}, 2 \mathrm{H}), 6.85-6.88(\mathrm{~m}, 2 \mathrm{H}), 4.67(\mathrm{dd}, 1 \mathrm{H}, J=7.6,7.6 \mathrm{~Hz}), 3.49(\mathrm{dd}, 1 \mathrm{H}, J$ $=8.0,14.0 \mathrm{~Hz}), 3.33(\mathrm{dd}, 1 \mathrm{H}, J=7.2,14.0 \mathrm{~Hz}), 2.34(\mathrm{~s}, 3 \mathrm{H}) ;{ }^{13} \mathrm{C}$ NMR $(100 \mathrm{MHz}$, $\left.\mathrm{CDCl}_{3}\right) \delta 167.9,148.0,136.0,135.6,130.0,129.5,128.7,127.5,121.2,120.7,57.1,41.1$, 20.8; IR ( $\mathrm{NaCl}$, neat) 1763, 1507, 1195, 1166, $1138 \mathrm{~cm}^{-1}$; HRMS (EI+) calcd for $\mathrm{C}_{16} \mathrm{H}_{15} \mathrm{ClO}_{2}, 274.0761$. Found 274.0760.

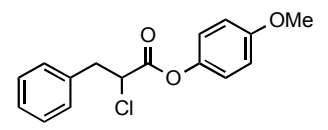

2-Chloro-3-phenyl-propionic acid 4-methoxy-phenyl ester: $\mathrm{Rf}=$ $0.48(3: 1 \mathrm{Hex} / \mathrm{EtOAc}) ;[\alpha]_{\mathrm{D}}^{24}=+23.4\left(\mathrm{c}=2.8, \mathrm{CHCl}_{3}\right) ;$ HPLC analysis - Chiracel AD-H column $97: 3$ hexanes : isopropanol $1 \mathrm{~mL}$ / min. Major: 11.6 minutes, Minor: 10.8 minutes. ${ }^{1} \mathrm{H}$ NMR $\left(400 \mathrm{MHz}, \mathrm{CDCl}_{3}\right) \delta 7.15-$ $7.39(\mathrm{~m}, 6 \mathrm{H}), 6.86-6.91(\mathrm{~m}, 3 \mathrm{H}), 4.66(\mathrm{dd}, 1 \mathrm{H}, J=8.1,8.1 \mathrm{~Hz}), 3.79(\mathrm{~s}, 3 \mathrm{H}), 3.48$ (dd, $1 \mathrm{H}, J=7.9,13.8 \mathrm{~Hz}), 3.32(\mathrm{dd}, 1 \mathrm{H}, J=7.2,13.8 \mathrm{~Hz}) ;{ }^{13} \mathrm{C} \mathrm{NMR}\left(100 \mathrm{MHz}, \mathrm{CDCl}_{3}\right) \delta$ 168.1, 157.6, 143.7, 135.6, 129.5, 128.7, 127.5, 121.8, 114.5, 57.0, 55.6, 41.2; IR (NaCl, neat) $1763,1505,1248,1191,1139 \mathrm{~cm}^{-1}$; HRMS (FAB+) calcd for $\mathrm{C}_{16} \mathrm{H}_{15} \mathrm{ClO}_{3}$, 290.0710. Found 290.0718.

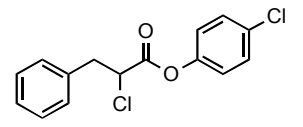

2-Chloro-3-phenyl-propionic acid 4-chloro-phenyl ester: $\mathrm{Rf}=0.85$ $(3: 1 \mathrm{Hex} / \mathrm{EtOAc}) ;[\alpha]_{\mathrm{D}}{ }^{24}=+35.1\left(\mathrm{c}=1.6, \mathrm{CHCl}_{3}\right) ; \mathrm{HPLC}$ analysis Chiracel OD-H column 99 : 1 hexanes : isopropanol $0.3 \mathrm{~mL} / \mathrm{min}$. Major: 32.1 minutes, Minor: 34.2 minutes. ${ }^{1} \mathrm{H} \mathrm{NMR}\left(400 \mathrm{MHz}, \mathrm{CDCl}_{3}\right) \delta$ 7.25-7.38 (m, 7H), 7.18-7.20 (m, 1H), 6.89-6.92 (m, 2H), 4.67 (ddd, 1H, J = 0.6, 7.2, 7.2 Hz), 3.47 (dd, $1 \mathrm{H}, J=8.4,14.0 \mathrm{~Hz}), 3.37(\mathrm{dd}, 1 \mathrm{H}, J=7.2,14 \mathrm{~Hz}), 2.34(\mathrm{~s}, 3 \mathrm{H}) ;{ }^{13} \mathrm{C} \mathrm{NMR}(100 \mathrm{MHz}$, $\left.\mathrm{CDCl}_{3}\right) \delta 167.6,148.6,135.4,131.8,129.6,129.4,128.8,127.6,122.5,56.8,41.1$; IR $\left(\mathrm{NaCl}\right.$, neat) $1764,1486,1197,1136,1090 \mathrm{~cm}^{-1}$; HRMS (EI+) calcd for $\mathrm{C}_{15} \mathrm{H}_{12} \mathrm{Cl}_{2} \mathrm{O}_{2}$, 294.0214. Found 294.0220.

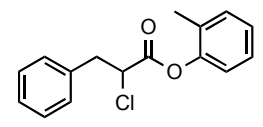

2-Chloro-3-phenyl-propionic acid $o$-tolyl ester: $\quad \mathrm{Rf}=0.61(3: 1$ $\mathrm{Hex} / \mathrm{EtOAc}) ;[\alpha]_{\mathrm{D}}{ }^{24}=+13.5\left(\mathrm{c}=2.1, \mathrm{CHCl}_{3}\right) ;$ HPLC analysis Chiracel OB-H column 99 : 1 hexanes : isopropanol $0.3 \mathrm{~mL} / \mathrm{min}$. Major: 39.9 minutes, Minor: 42.4 minutes. ${ }^{1} \mathrm{H} \mathrm{NMR}\left(400 \mathrm{MHz}, \mathrm{CDCl}_{3}\right) \delta$ 7.29-7.38 (m, $5 \mathrm{H}), 7.13-7.22(\mathrm{~m}, 5 \mathrm{H}), 6.86(\mathrm{dd}, 1 \mathrm{H}, J=1.7,7.5 \mathrm{~Hz}), 4.7(\mathrm{dd}, 1 \mathrm{H}, J=7.2,7.9 \mathrm{~Hz}), 3.52$ $(\mathrm{dd}, 1 \mathrm{H}, J=8.1,14.1 \mathrm{~Hz}), 3.33(\mathrm{dd}, 1 \mathrm{H}, J=7.3,13.9 \mathrm{~Hz}), 2.06(\mathrm{~s}, 3 \mathrm{H}) ;{ }^{13} \mathrm{C}$ NMR $(100$ 
$\left.\mathrm{MHz}, \mathrm{CDCl}_{3}\right) \delta 167.5,148.8,135.6,131.3,130.0,129.5,128.7,127.5,126.9,126.5$, 121.3, 56.8, 41.0, 15.8; IR ( $\mathrm{NaCl}$, neat) 1765, 1490, 1222, 1171, 1141, $1109 \mathrm{~cm}^{-1}$; HRMS (FAB+) calcd for $\mathrm{C}_{16} \mathrm{H}_{16} \mathrm{O}_{2} \mathrm{Cl}, 275.0838$. Found 275.0830.

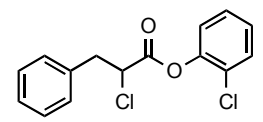

2-Chloro-3-phenyl-propionic acid 2-chloro-phenyl ester: $\mathrm{Rf}=0.61$ $(3: 1 \mathrm{Hex} / \mathrm{EtOAc}) ;[\alpha]_{\mathrm{D}}{ }^{24}=+8.1\left(\mathrm{c}=2.5, \mathrm{CHCl}_{3}\right) ;$ HPLC analysis Chiracel OB-H column 97 : 3 hexanes : isopropanol $0.3 \mathrm{~mL} / \mathrm{min}$. Major: 34.4 minutes, Minor: 36.5 minutes. ${ }^{1} \mathrm{H} \mathrm{NMR}\left(400 \mathrm{MHz}, \mathrm{CDCl}_{3}\right) \delta$ 7.43-7.45 (m, $1 \mathrm{H}), 7.20-7.39(\mathrm{~m}, 7 \mathrm{H}), 7.01-7.04(\mathrm{~m}, 1 \mathrm{H}), 4.75(\mathrm{dd}, 1 \mathrm{H}, J=7.6,7.6 \mathrm{~Hz}), 3.57(\mathrm{dd}, 1 \mathrm{H}, J$ $=7.2,14.0 \mathrm{~Hz}), 3.34(\mathrm{dd}, 1 \mathrm{H}, J=7.6,14.0 \mathrm{~Hz}) ;{ }^{13} \mathrm{C} \mathrm{NMR}\left(100 \mathrm{MHz}, \mathrm{CDCl}_{3}\right) \delta 166.9$, $146.4,135.5,130.5,129.5,128.7,127.8,127.5,126.7,123.2,56.9,41.0 ;$ IR (NaCl, neat) 1773, 1476, 1213, 1137, $1124 \mathrm{~cm}^{-1}$; HRMS (FAB+) calcd for $\mathrm{C}_{15} \mathrm{H}_{13} \mathrm{Cl}_{2} \mathrm{O}_{2}$, Calcd 295.0293 Found 295.0284.

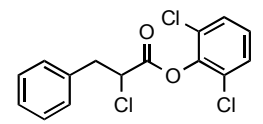

2-Chloro-3-phenyl-propionic acid 2,6-dichloro-phenyl ester: $\mathrm{Rf}=$ $0.58(3: 1 \mathrm{Hex} / \mathrm{EtOAc}) ;[\alpha]_{\mathrm{D}}{ }^{24}=-1.0\left(\mathrm{c}=2.2, \mathrm{CHCl}_{3}\right) ; \mathrm{HPLC}$ analysis performed on corresponding alcohol provided by LAH reduction Chiracel ASH 90 : 10 hexanes : isopropanol $0.5 \mathrm{~mL} / \mathrm{min}$. Major: 13.0 minutes, Minor: 14.8 minutes. ${ }^{1} \mathrm{H}$ NMR $\left(400 \mathrm{MHz}, \mathrm{CDCl}_{3}\right) \delta$ 7.30-7.40 (m, 7H), 7.14-7.18 (m, 1H), 4.83 $(\mathrm{dd}, 1 \mathrm{H}, J=6.8,8.4 \mathrm{~Hz}), 3.65(\mathrm{dd}, 1 \mathrm{H}, J=6.8,14.4 \mathrm{~Hz}), 3.35(\mathrm{dd}, 1 \mathrm{H}, J=8.0,14.0 \mathrm{~Hz})$; ${ }^{13} \mathrm{C}$ NMR $\left(100 \mathrm{MHz} \mathrm{CDCl}_{3}\right) \delta 165.7,143.2,135.4,129.5,128.7,128.6,127.6,127.4$, 56.5, 40.8; IR ( NaCl, neat) 1773, 1445, 1227, $1127 \mathrm{~cm}^{-1}$; HRMS (FAB+) calcd for $\mathrm{C}_{15} \mathrm{H}_{12} \mathrm{Cl}_{3} \mathrm{O}_{2}, 328.9903$. Found 328.9906 .

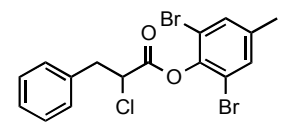

2-Chloro-3-phenyl-propionic acid 2,6-dibromo-4-methyl-phenyl ester: $\mathrm{Rf}=0.68(3: 1 \mathrm{Hex} / \mathrm{EtOAc}) ;[\alpha]_{\mathrm{D}}{ }^{24}=-2.8\left(\mathrm{c}=3.0, \mathrm{CHCl}_{3}\right)$; HPLC analysis - Chiracel AD-H column $97: 3$ hexanes : isopropanol 1 $\mathrm{mL} /$ min. Major: 6.2 minutes, Minor: 7.3 minutes. ${ }^{1} \mathrm{H} \mathrm{NMR}\left(400 \mathrm{MHz}, \mathrm{CDCl}_{3}\right) \delta 7.27$ $7.33(\mathrm{~m}, 7 \mathrm{H}), 4.76(\mathrm{dd}, 1 \mathrm{H}, J=7.0 \mathrm{~Hz}), 3.62(\mathrm{dd}, 1 \mathrm{H}, J=6.4,14.1 \mathrm{~Hz}), 3.29(\mathrm{dd}, 1 \mathrm{H}, J=$ 8.7, $14.1 \mathrm{~Hz}), 2.30(\mathrm{~s}, 3 \mathrm{H}) ;{ }^{13} \mathrm{C} \mathrm{NMR}\left(100 \mathrm{MHz}, \mathrm{CDCl}_{3}\right) \delta 165.8,143.1,139.2,135.6$, 132.9, 129.6, 128.7, 127.4, 116.7, 56.8, 40.8, 20.4; IR ( NaCl, neat) 1782, 1457, 1243, $1196,1124 \mathrm{~cm}^{-1}$; HRMS (FAB+) calcd for $\mathrm{C}_{16} \mathrm{H}_{13} \mathrm{ClO}_{2} \mathrm{Br}_{2}$, 429.8971. Found 429.8968 .

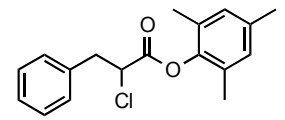

2-Chloro-3-phenyl-propionic acid 2,4,6-trimethyl-phenyl ester: Rf $=0.61(3: 1 \mathrm{Hex} / \mathrm{EtOAc}) ;{ }^{1} \mathrm{H}$ NMR $\left(400 \mathrm{MHz}, \mathrm{CDCl}_{3}\right) \delta 7.29-7.38(\mathrm{~m}$, $5 \mathrm{H}), 6.85-6.87(\mathrm{~m}, 2 \mathrm{H}), 4.75(\mathrm{dd}, 1 \mathrm{H}, J=7.2,8.4 \mathrm{~Hz}), 3.55(\mathrm{dd}, 1 \mathrm{H}, J$ $=8.4,14.0 \mathrm{~Hz}), 3.33(\mathrm{dd}, 1 \mathrm{H}, J=7.2,14.0 \mathrm{~Hz}), 2.27(\mathrm{~s}, 3 \mathrm{H}), 1.95(\mathrm{~s}, 6 \mathrm{H}) ;{ }^{13} \mathrm{C} \mathrm{NMR}$ $\left(100 \mathrm{MHz}, \mathrm{CDCl}_{3}\right) \delta 167.2,145.3,135.8,135.7,131.1,129.5,129.3,128.7,127.4,56.4$, 40.8, 20.7, 15.8; IR (NaCl, neat) 1760, 1191, $1144 \mathrm{~cm}^{-1}$; HRMS (EI+) calcd for $\mathrm{C}_{18} \mathrm{H}_{19} \mathrm{ClO}_{2}, 302.1074$. Found 302.1076.

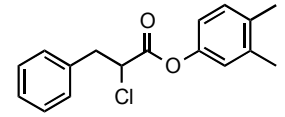

2-Chloro-3-phenyl-propionic acid 3,4-dimethyl-phenyl ester: $\mathrm{Rf}=$ $0.70(3: 1 \mathrm{Hex} / \mathrm{EtOAc}) ;[\alpha]_{\mathrm{D}}{ }^{24}=+21.6\left(\mathrm{c}=2.9, \mathrm{CHCl}_{3}\right) ; \mathrm{HPLC}$ analysis - Chiracel AD-H column $97: 3$ hexanes : isopropanol $1 \mathrm{~mL} /$ min. Major: 6.5 minutes, Minor: 6.0 minutes. ${ }^{1} \mathrm{H}$ NMR $\left(300 \mathrm{MHz}, \mathrm{CDCl}_{3}\right) \delta$ 7.31-7.38 $(\mathrm{m}, 5 \mathrm{H}), 7.12(\mathrm{~d}, 1 \mathrm{H}, J=8.1 \mathrm{~Hz}), 6.70-6.75(\mathrm{~m}, 2 \mathrm{H}), 4.67(\mathrm{dd}, 1 \mathrm{H}, J=7.7,7.7 \mathrm{~Hz}), 3.50$ 
$(\mathrm{dd}, 1 \mathrm{H}, J=7.7,13.9 \mathrm{~Hz}), 3.33(\mathrm{dd}, 1 \mathrm{H}, J=7.3,13.9 \mathrm{~Hz}), 2.25(\mathrm{~s}, 6 \mathrm{H}) ;{ }^{13} \mathrm{C} \mathrm{NMR}(75$ $\left.\mathrm{MHz} \mathrm{CDCl}_{3}\right) \delta 167.8,148.0,137.9,135.5,134.5,130.2,129.4,128.6,127.4,121.8$, 118.0, 57.1, 41.2, 19.9, 19.3; IR (NaCl, neat) 1763, 1497, 1454, 1241, $1155 \mathrm{~cm}^{-1}$; HRMS (EI+) calcd for $\mathrm{C}_{17} \mathrm{H}_{17} \mathrm{ClO}_{2}, 288.0917$. Found 288.0924.

\section{Determination of absolute configuration:}

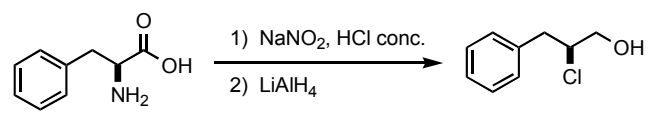

L-Phenylalanine was converted to (S)-2-chloro-3-phenyl-propanol according to literature procedures. $^{2}$ The HPLC trace of (S)-2-chloro-3-phenyl-propanol provided the same major enantiomer as the alcohol derived from 2-Chloro-3-phenyl-propionic acid 2,6dichloro-phenyl ester. 

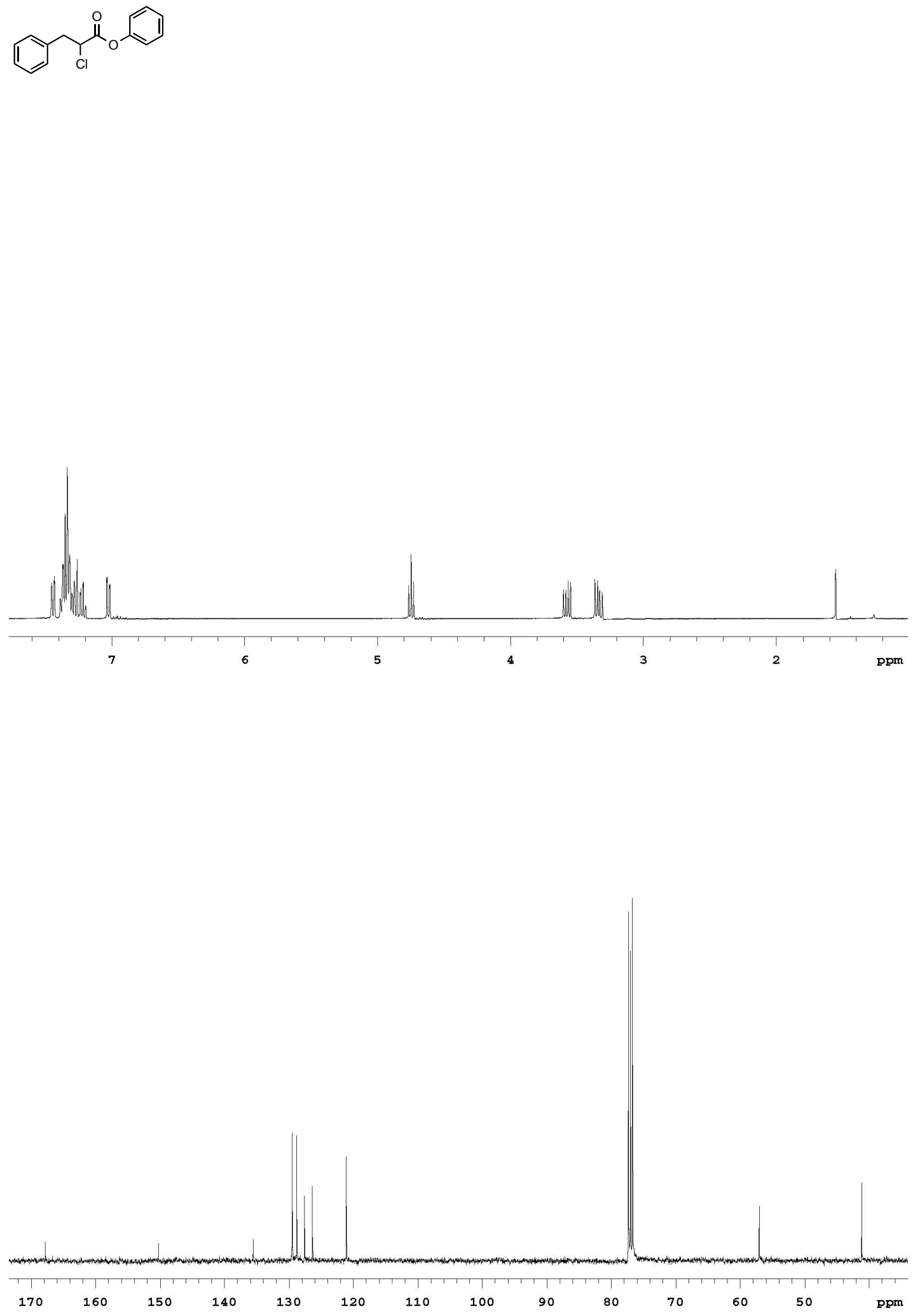

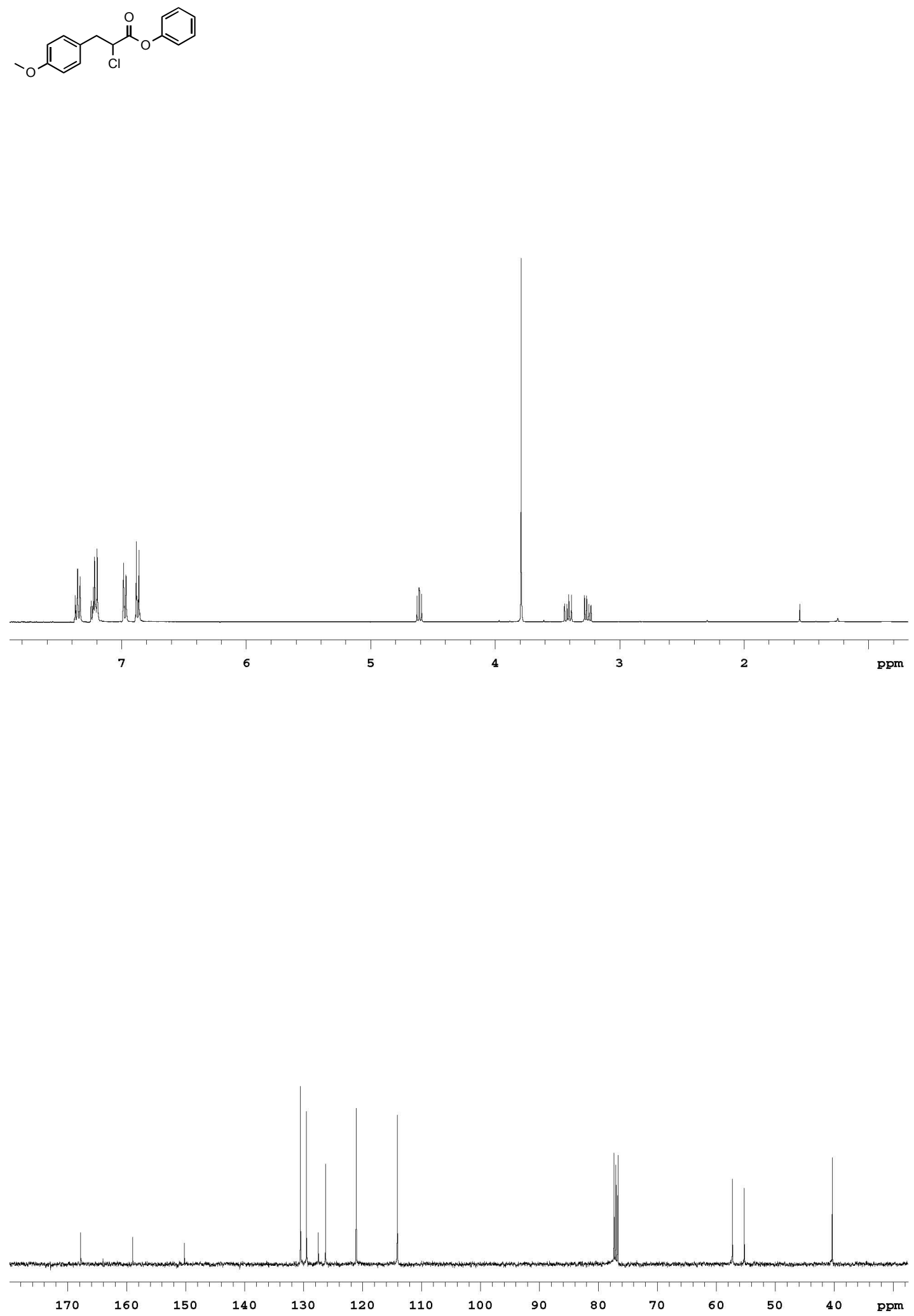
<smiles>O=C(Oc1ccccc1)C(Cl)CCc1ccccc1</smiles>
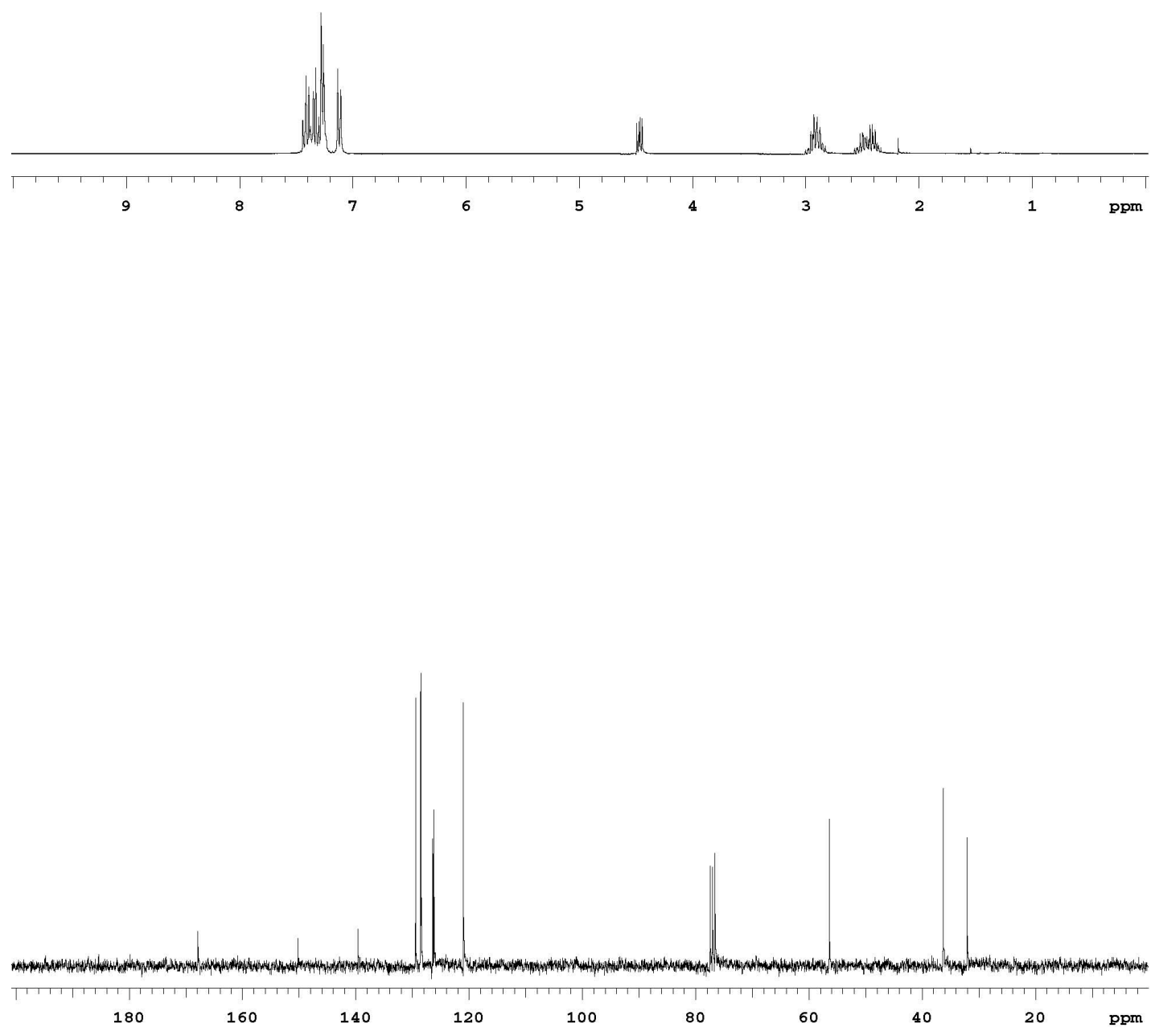
<smiles>O=C(Oc1ccccc1)C(Cl)CCCc1ccccc1</smiles>
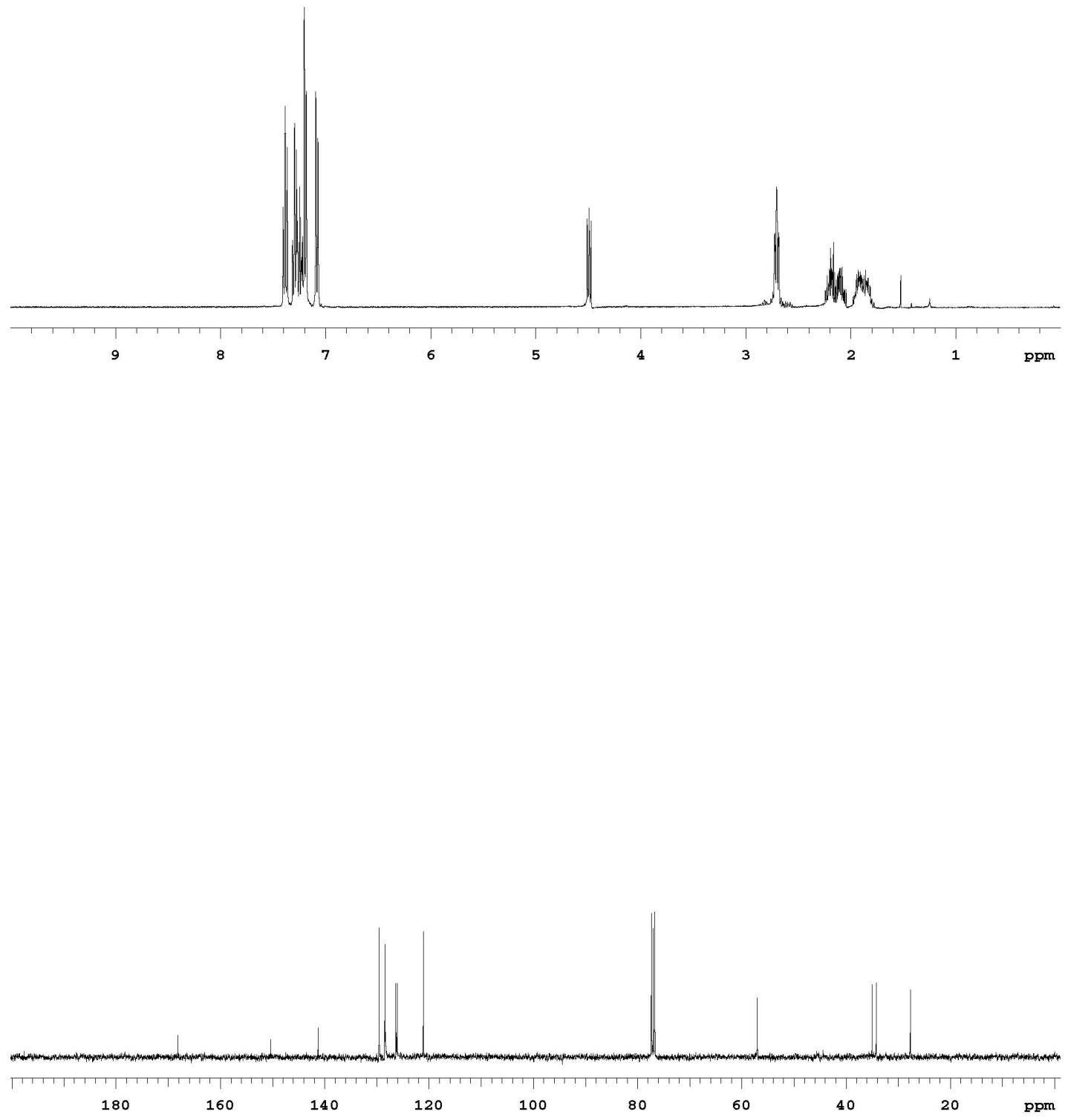

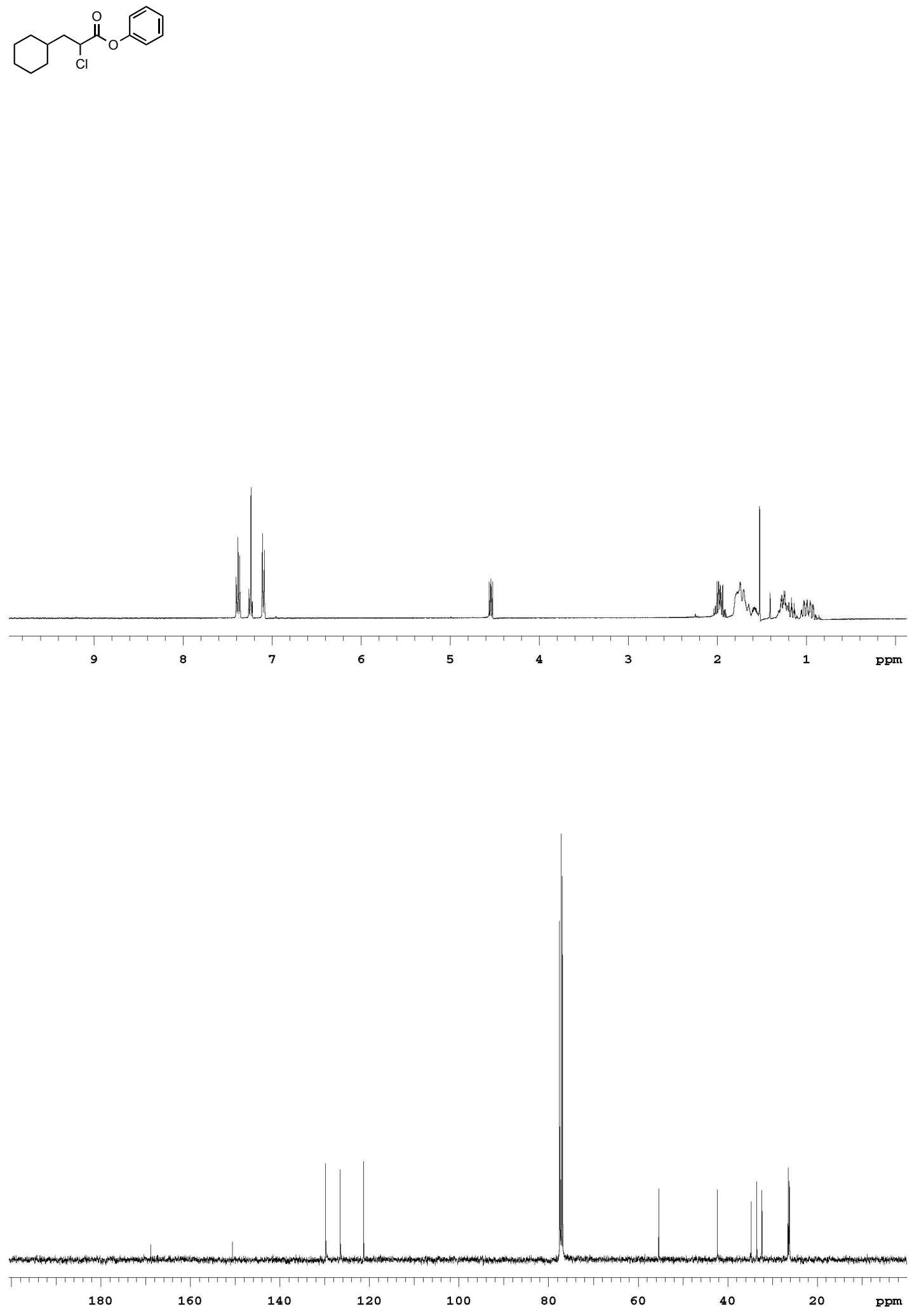

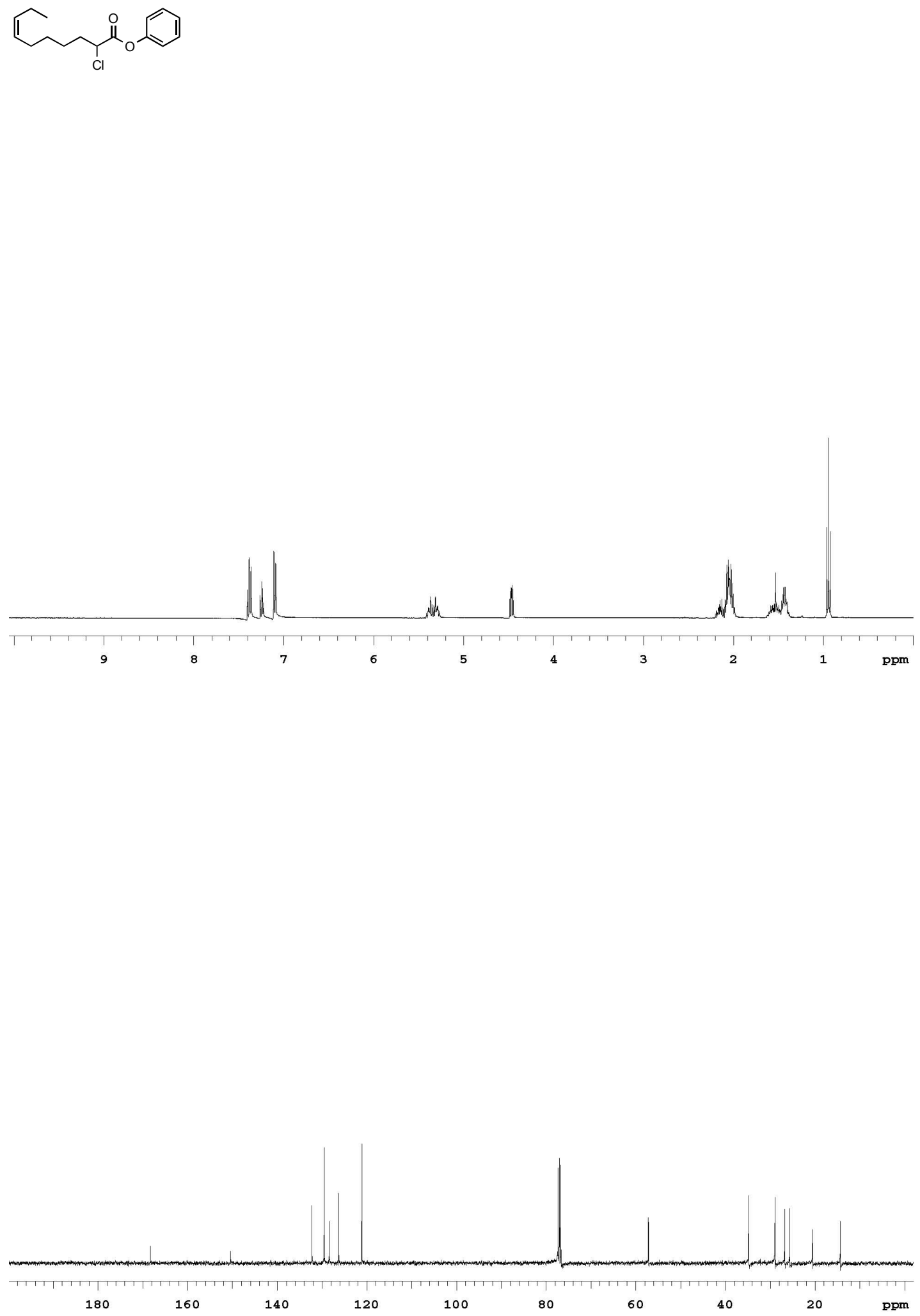

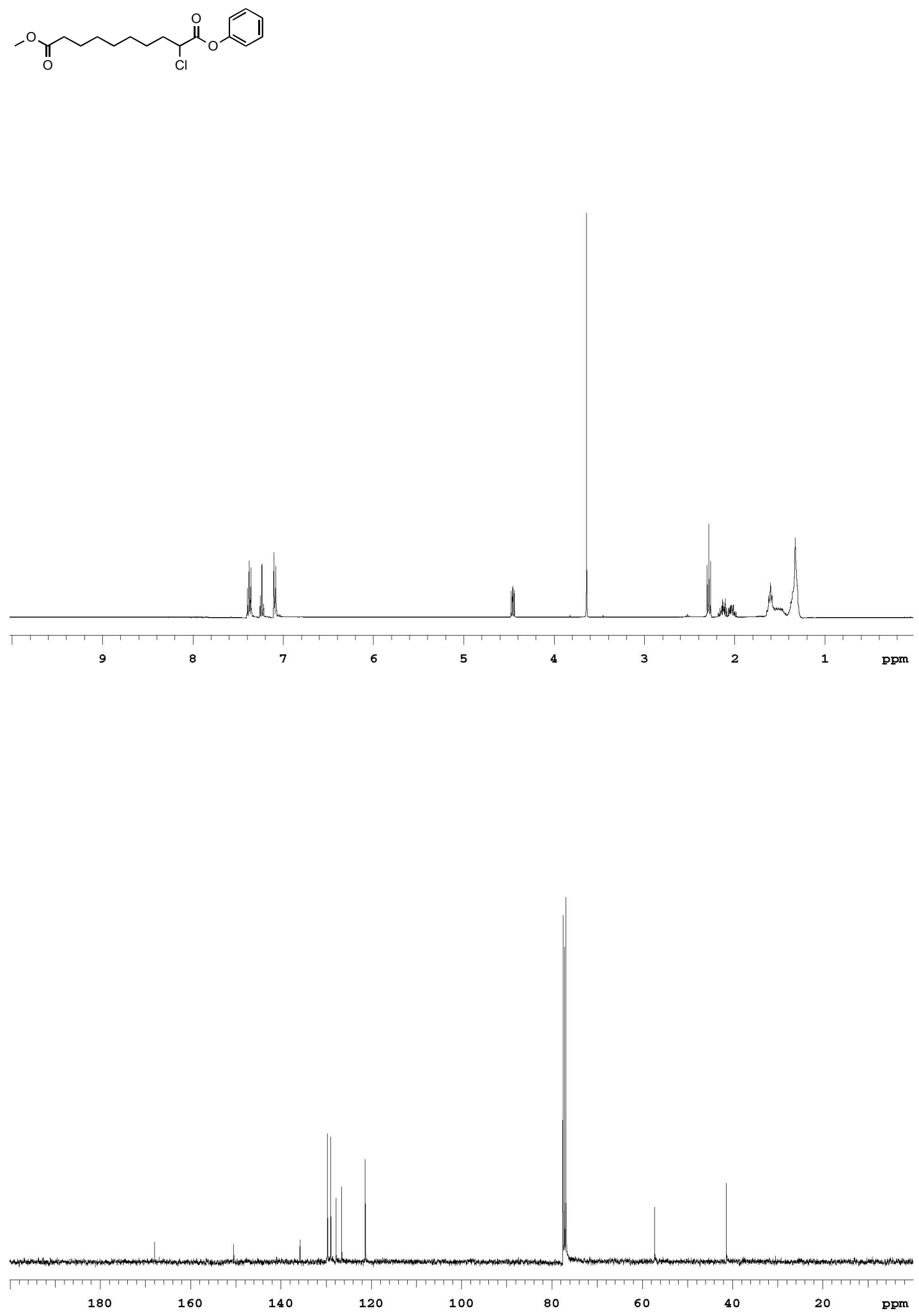

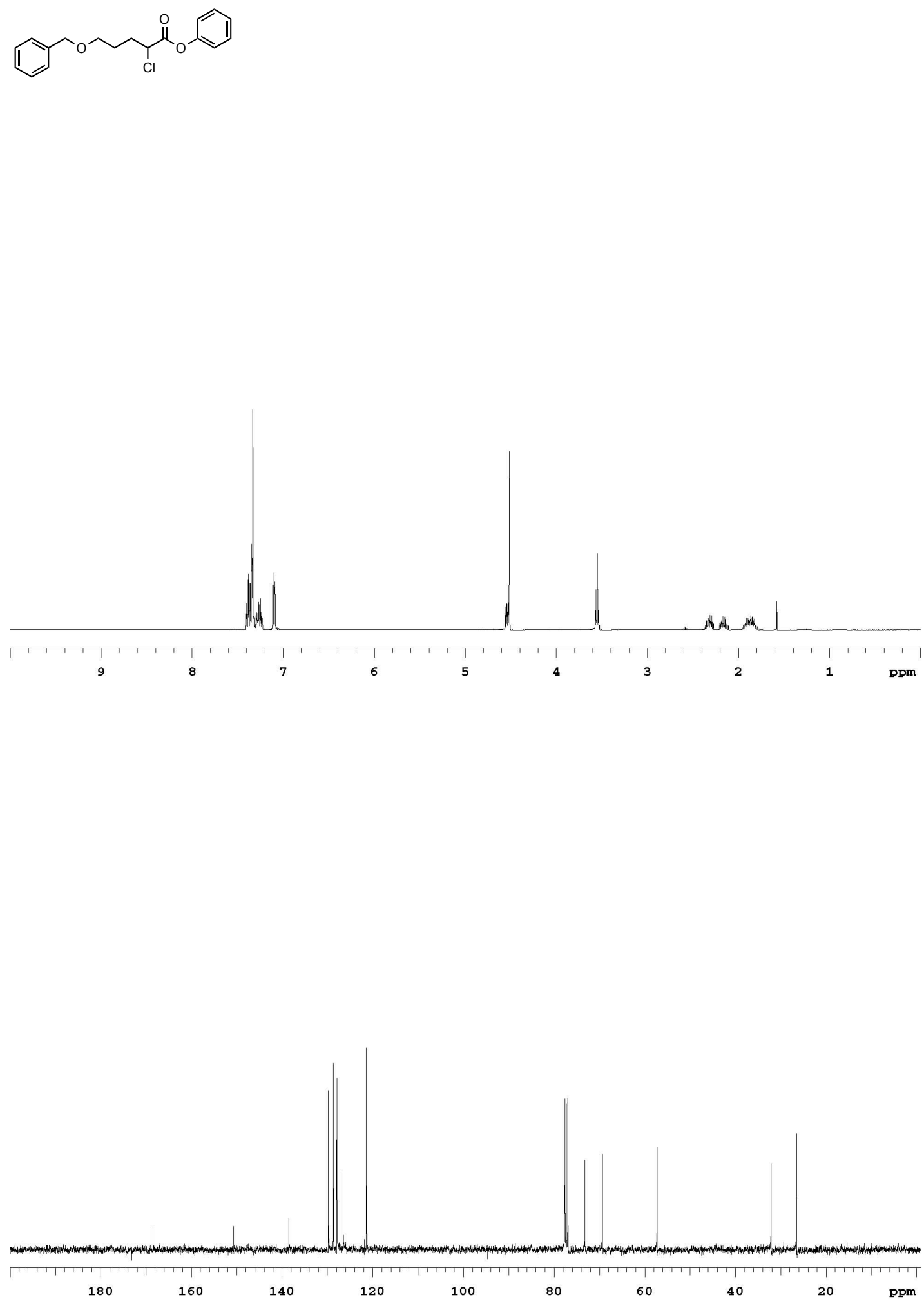
<smiles>CCCC/C=C\CC(Cl)C(=O)Oc1ccccc1</smiles>
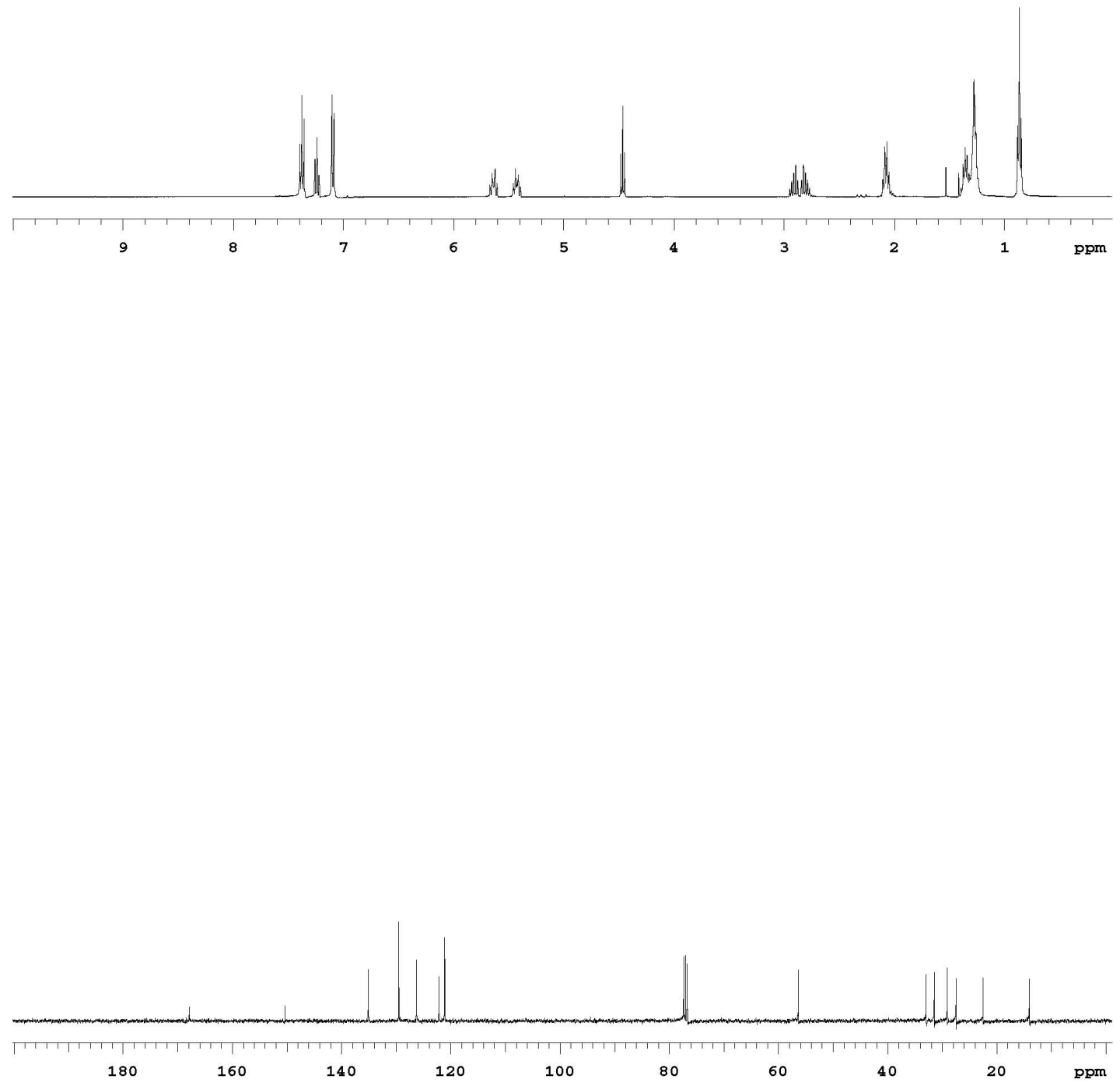

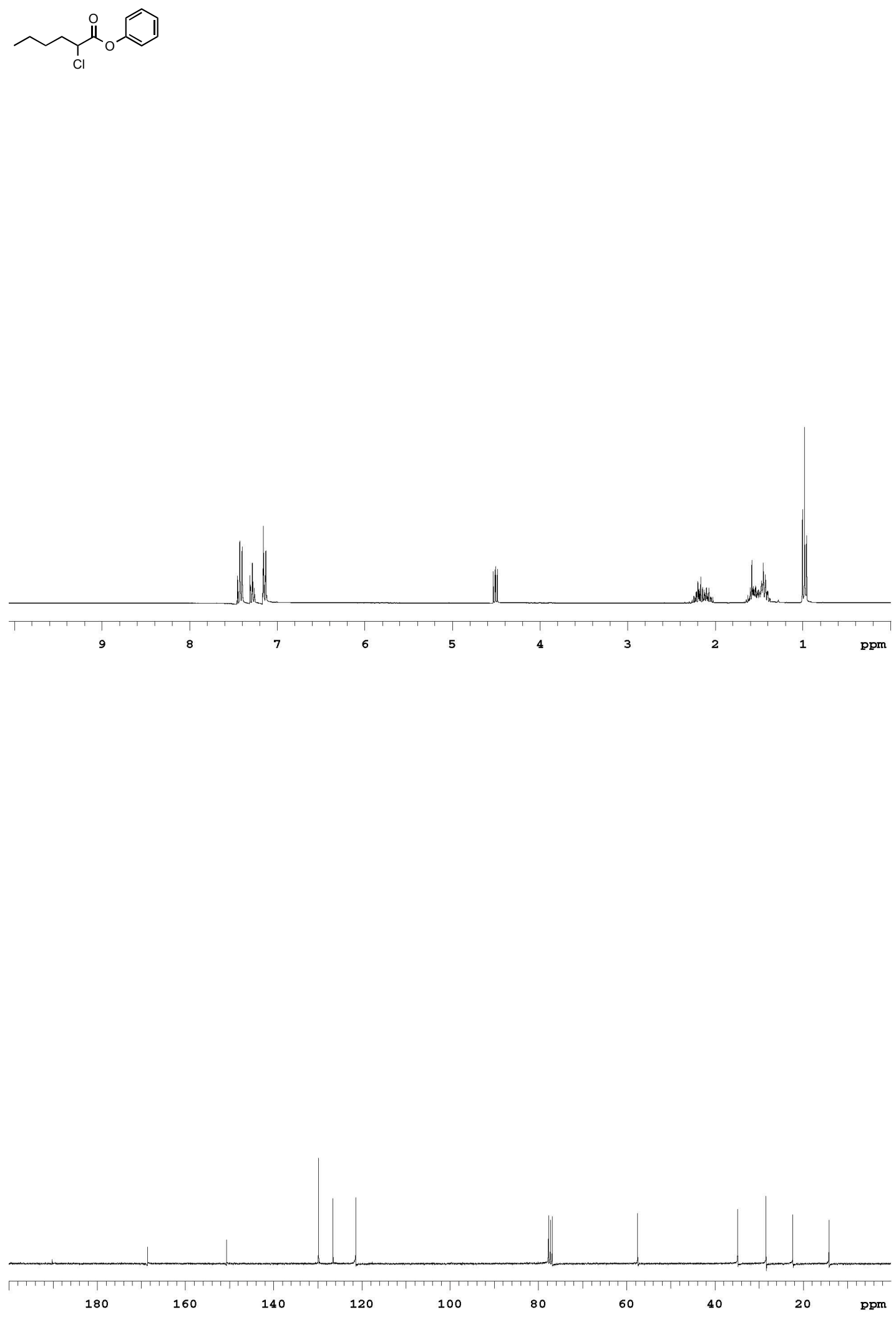

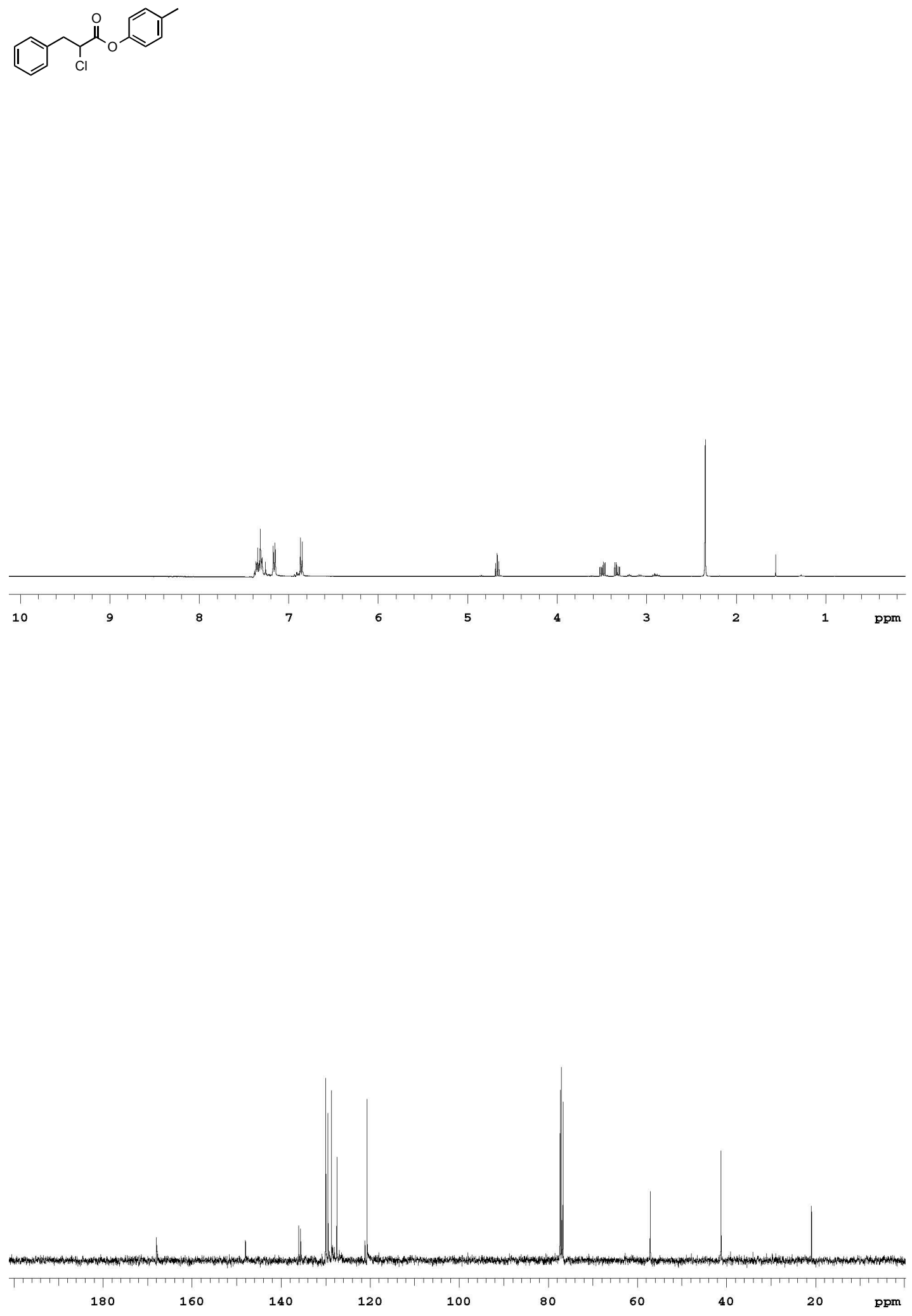
<smiles>COc1ccc(OC(=O)C(Cl)Cc2ccccc2)cc1</smiles>
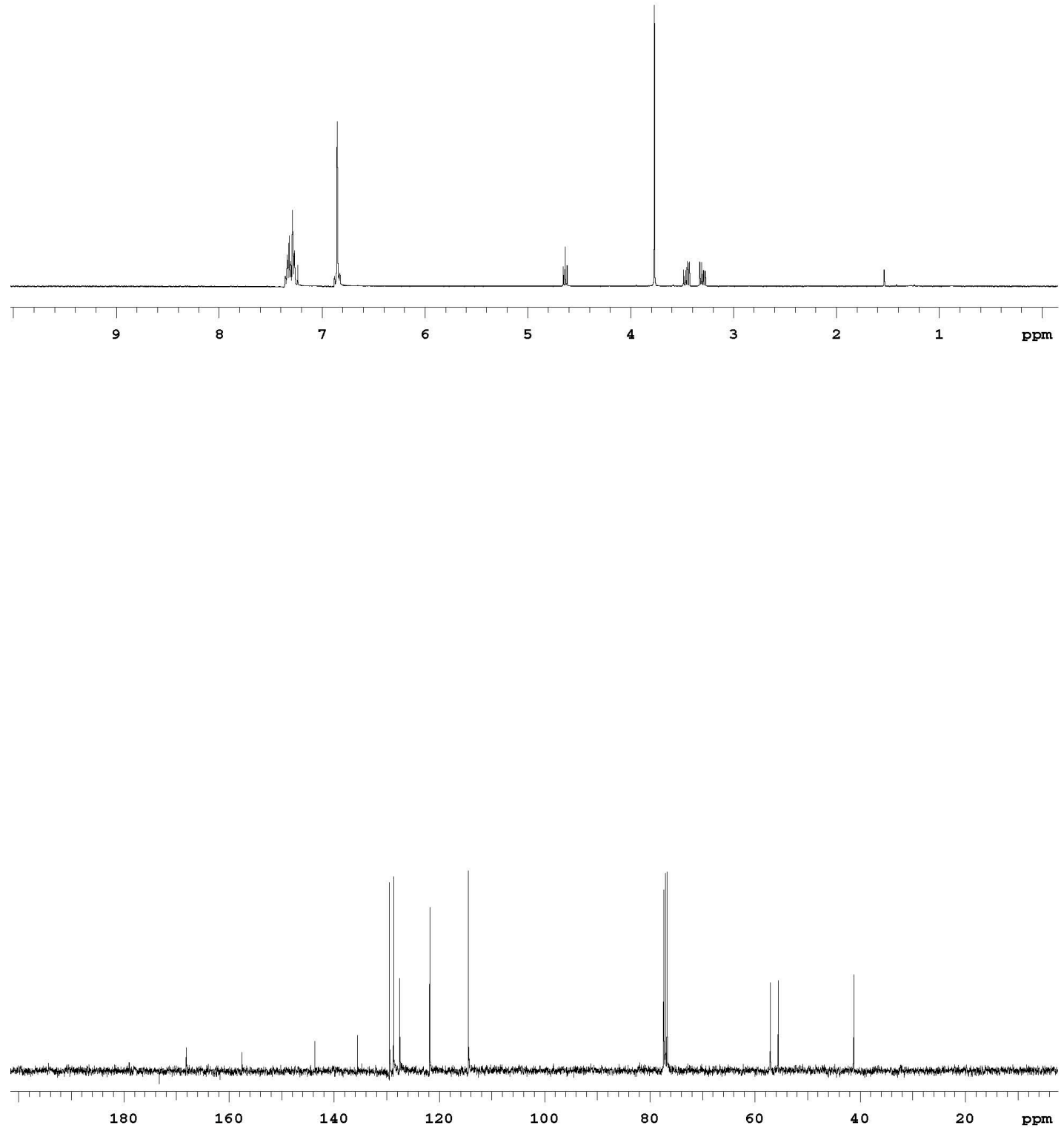

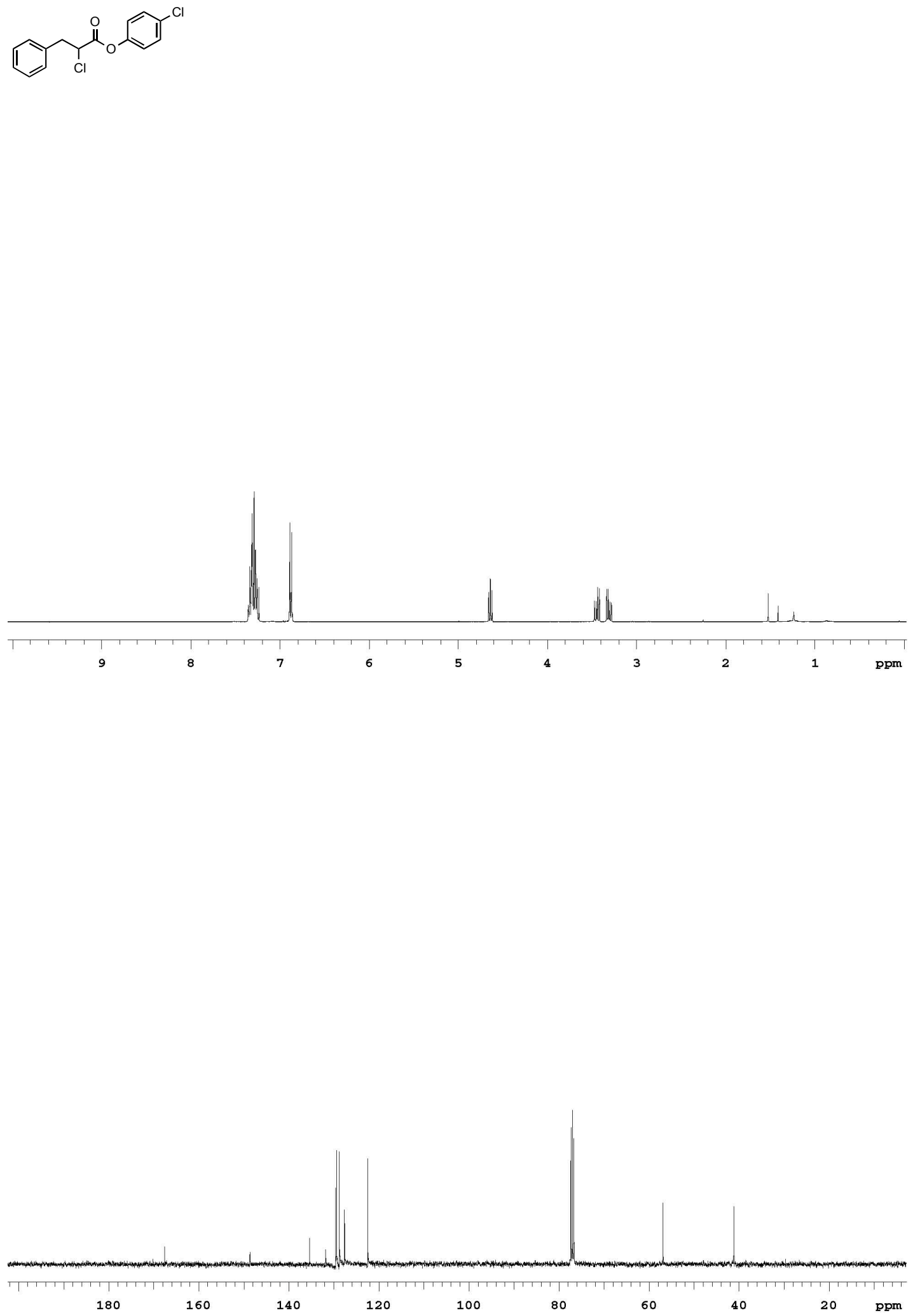

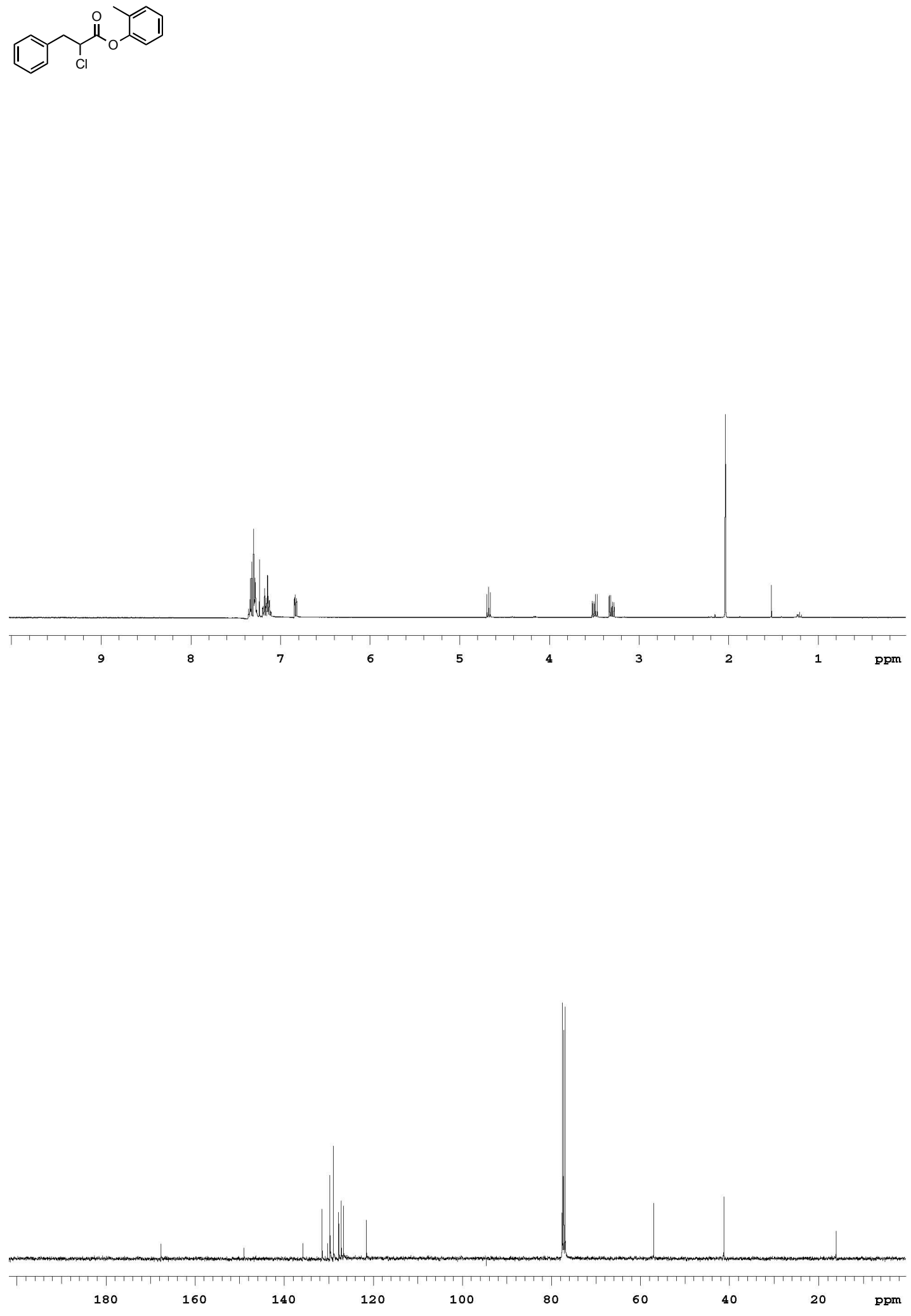

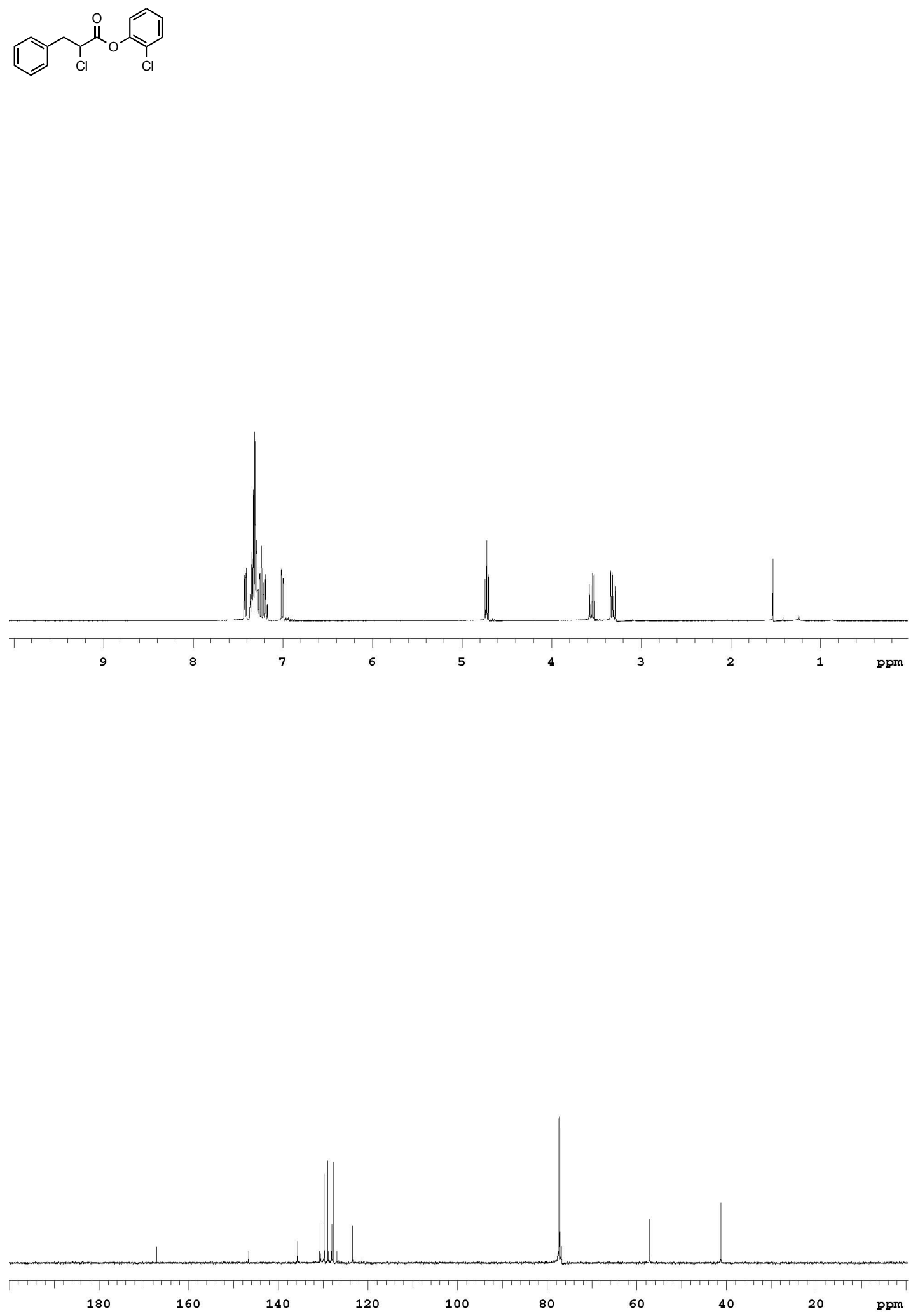
<smiles>O=C(Oc1c(Cl)cccc1Cl)C(Cl)Cc1ccccc1</smiles>
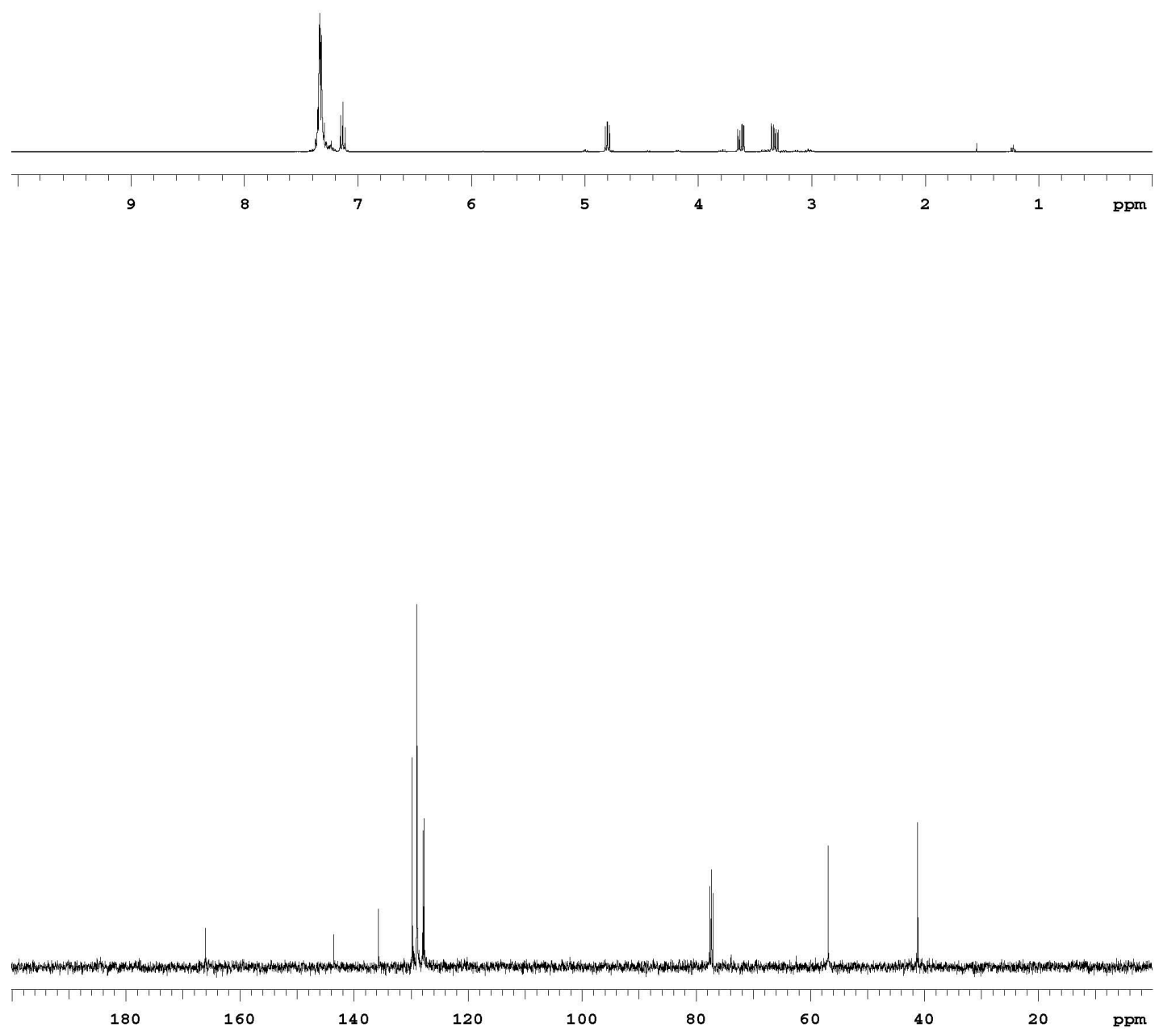

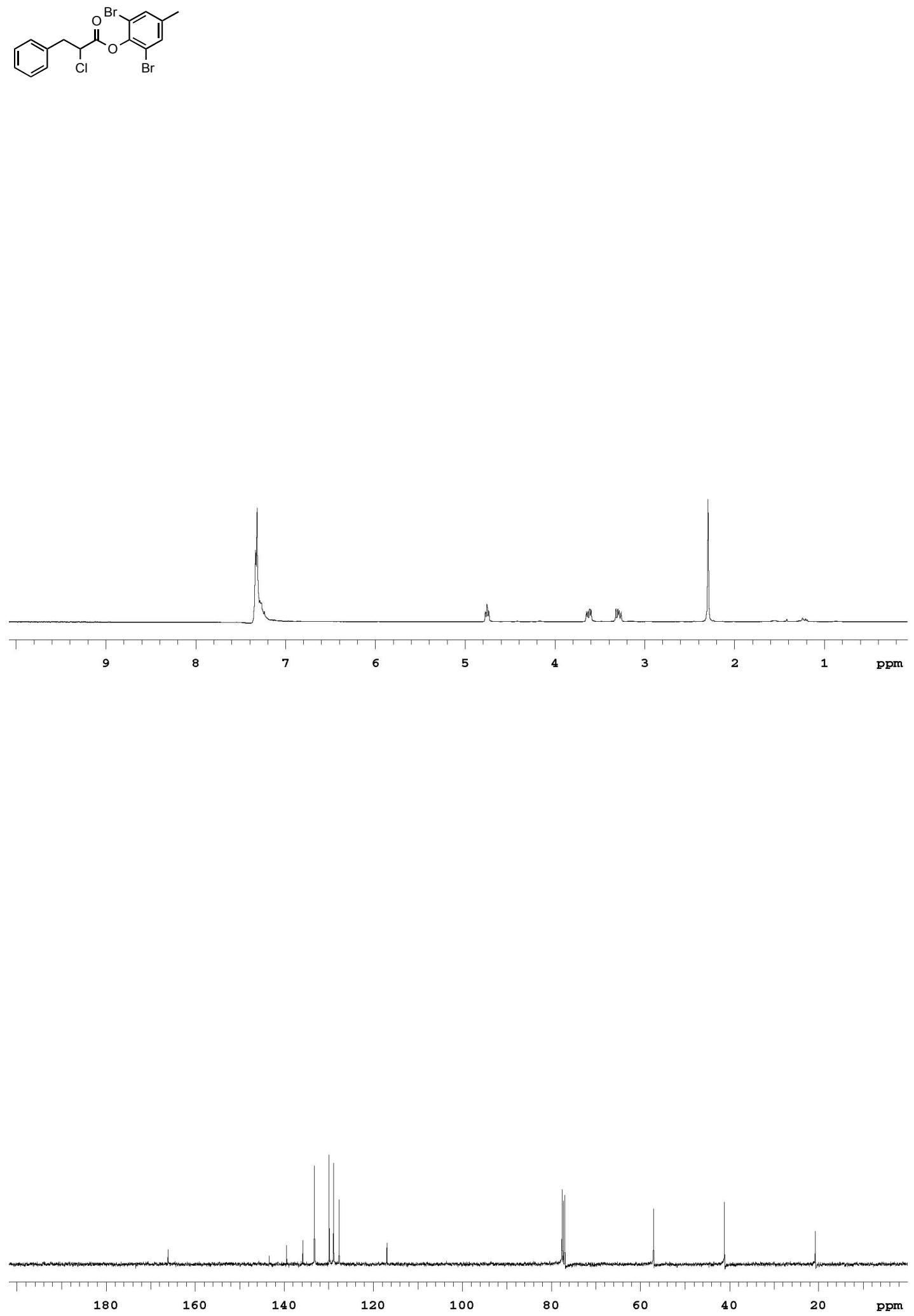

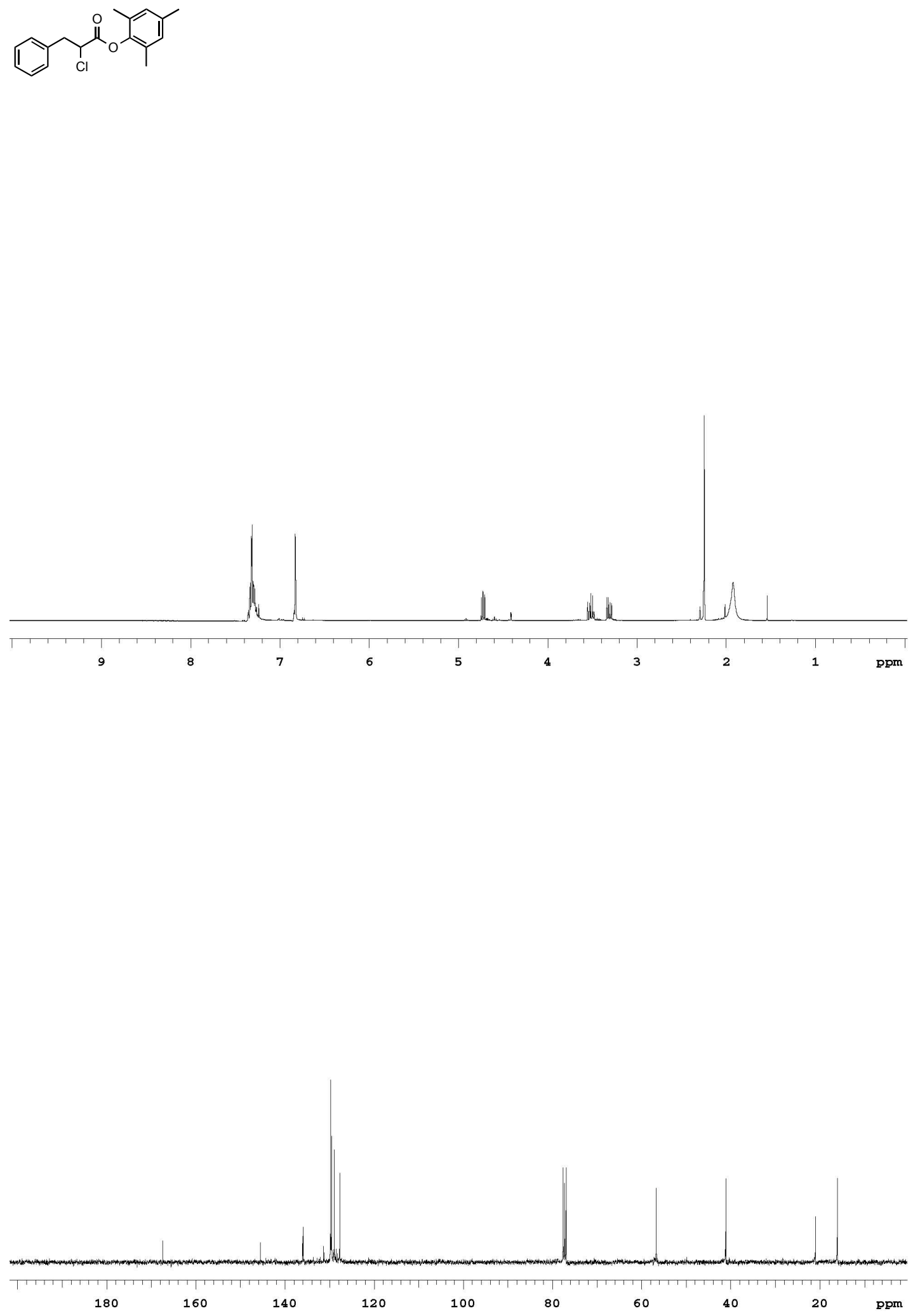

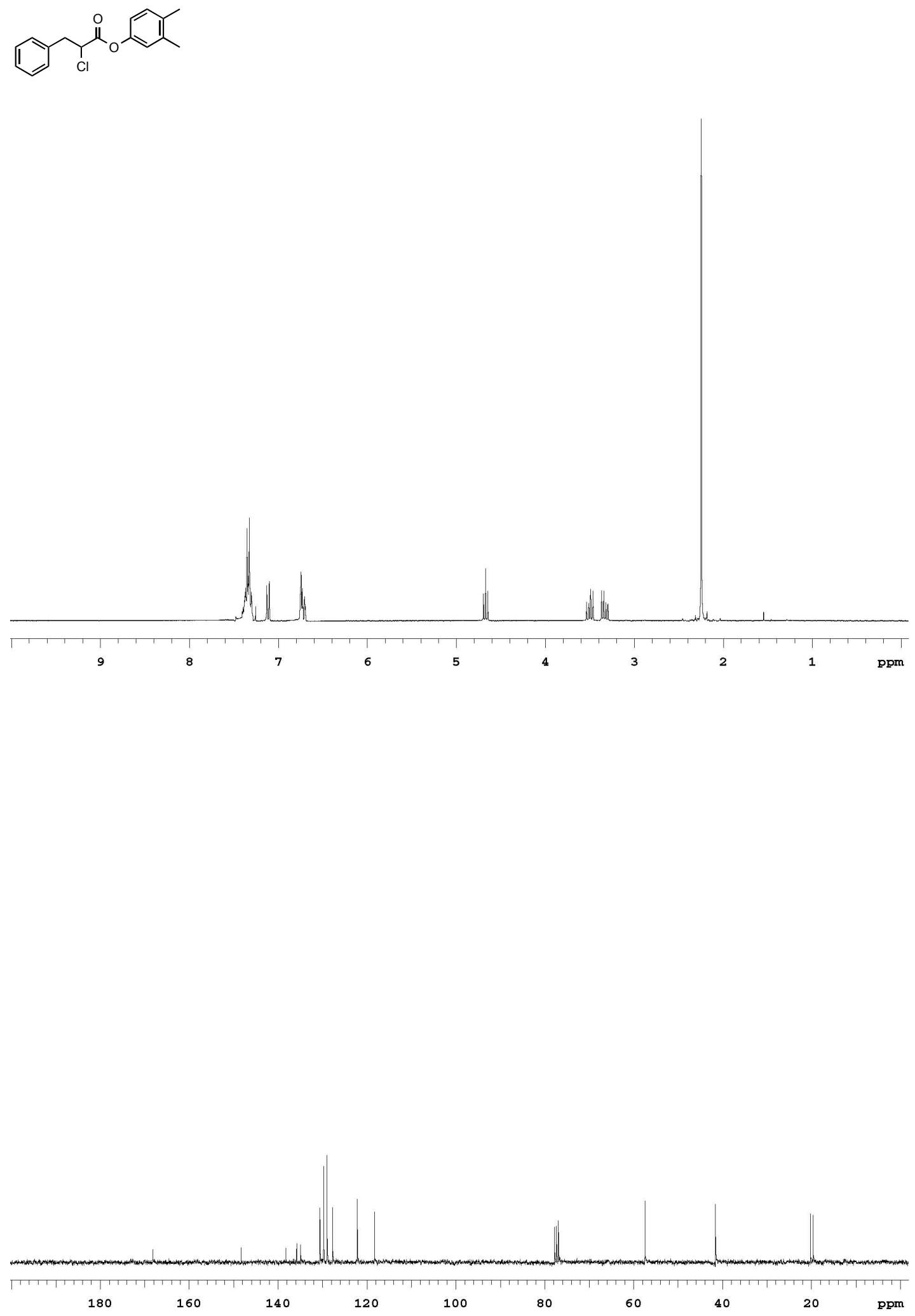

${ }^{1}$ Verhe, R.; De Kimpe, N.; De Buyck, L.; Schamp, N. Syn. Commun. 1975, 5, 455-456.

${ }^{2}$ Tan, E. W.; Chan, B.; Blackman, A. G. J. Am. Chem. Soc. 2002, 124, 2078-2079. 